\title{
The influence of scattering on the extinction of stars
}

\author{
E. Krügel
}

\author{
Max-Planck Institut für Radioastronomie, Auf dem Hügel 69, 53121 Bonn, Germany \\ e-mail: p309ekr@mpifr-bonn.mpg.de
}

Received 15 April 2008 / Accepted 2 October 2008

\begin{abstract}
Aims. In photometric measurements of stars, one usually assumes that all photons have reached the observer on a direct path and neglects the fraction that has been scattered. However, in observations of distant stars, for instance when they are in another galaxy, this fraction is not negligible. We investigate how scattered light enhances the flux and affects the extinction curve.

Methods. We compute the radiative transfer in a dusty medium using various techniques at wavelengths where dust emission is absent. Among the configurations considered are: a) homogeneous spheres with a central star; b) an infinite layer of stars behind or mixed with an infinite layer of dust which may be homogeneous or clumpy; c) one star in or behind a homogeneous or clumpy block of dust. In all cases, we define in analogy to the extinction optical depth $\tau$ an effective optical thickness $\tau^{\text {eff }}$ such that the flux attenuation is given by the factor $\exp \left(-\tau^{\mathrm{eff}}\right)$.

Results. a) We compute $\tau^{\text {eff }}$ and analyze how it depends, in different geometries, on the extinction optical depth, the dust albedo, the scattering phase function and the spatial resolution of the measurement; b) we calculate effective extinction curves towards single stars in clumpy and homogeneous media adopting standard optical dust properties. They show marked differences to the standard reddening curve; c) when determining $\tau^{\text {eff }}$ for a collection of spatially unresolved stars, we find discrepancies with some of the results in the literature.

Conclusions. When one converts the apparent magnitude of a star or a star cluster into an absolute magnitude, scattered light should be taken into account whenever the spatial resolution with which the measurement was performed corresponds to a linear scale over which the scattering optical depth around the source is greater than the relative observational error. One may try to determine $\tau^{\text {eff }}$ by solving the radiative transfer for an assumed distribution of stars and dust, or search for representative calculations in the literature, for example in the figures of this paper.
\end{abstract}

Key words. ISM: dust, extinction - radiative transfer

\section{Introduction}

At wavelengths where dust emission is negligible $(\lesssim 10 \mu \mathrm{m})$, the presence of dust usually dims the light of a star and reddens it. Photometric measurements yield the apparent fluxes of the star, but often allow, given reasonable assumptions, also the determination of its intrinsic luminosity. In the standard scenario, where the dust lies somewhere between the Sun and the star and the aperture employed in the observations is small, the observer does not detect any scattered light. The change in color is then described by the interstellar extinction or reddening curve, $T_{\lambda}$, and the attenuation at wavelength $\lambda$ by the extinction optical depth $\tau_{\lambda}$ along the line of sight from the star to the observer.

However, it had long been realized that in the case of circumstellar shells, disks of stars permeated by a dusty medium, nebular emission, compact young stellar clusters and whole galaxies, an observer invariably also sees scattered light (Mathis 1972; Greenberg \& Wang 1972; Natta \& Panagia 1984; Bruzual et al. 1988; Calzetti et al. 1994, henceforth CKS; Voshchinnikov et al. 1996, Witt \& Gordon 2000; Fischera \& Dopita 2005). It has to be taken properly into account when one wants to convert the measured apparent fluxes into intrinsic ones. It is the purpose of the present paper to continue and extend these studies and to quantify, by numerical calculations, the contribution of the scattered light for a larger variety of geometrical configurations.

\section{The standard extinction curve}

The simplest and most physical way to define the interstellar extinction curve, $T_{\lambda}$, is by the ratio

$$
T_{\lambda}=\frac{K_{\lambda}^{\mathrm{ext}}}{K_{\mathrm{V}}^{\mathrm{ext}}}
$$

The extinction coefficient $K_{\lambda}^{\text {ext }}$ is equal to the sum of the absorption and scattering coefficient,

$K_{\lambda}^{\mathrm{ext}}=K_{\lambda}^{\mathrm{abs}}+K_{\lambda}^{\mathrm{sca}}$

$V$ is the visual band at $0.55 \mu \mathrm{m}$. Alternatively, $T_{\lambda}$ can be written as the ratio of extinction optical depths towards a star,

$T_{\lambda}=\frac{\tau_{\lambda}}{\tau_{\mathrm{V}}}$

As the interstellar extinction curve is fairly uniform in all directions of the sky, which suggests that dust properties themselves are fairly uniform, we will speak of the "standard curve" and "standard dust" and disregard here important deviations as they occur, for example, in dark clouds. 
When $T_{\lambda}$ is known everywhere, one can derive from it the extinction curve in its traditional form, $E_{\lambda}$, which is closely related to the procedure by which $E_{\lambda}$ is obtained,

$E_{\lambda}=\frac{A_{\lambda}-A_{\mathrm{V}}}{A_{\mathrm{B}}-A_{\mathrm{V}}}=\frac{\tau_{\lambda}-\tau_{\mathrm{V}}}{\tau_{\mathrm{B}}-\tau_{\mathrm{V}}}$.

$A_{\lambda}$ is the attenuation in magnitudes. At long wavelengths, $A_{\lambda}$ tends to zero and one defines the ratio of total-to-selective extinction by

$R_{\mathrm{V}}=-E_{\infty}=\frac{A_{\mathrm{V}}}{A_{\mathrm{B}}-A_{\mathrm{V}}}=\frac{A_{\mathrm{V}}}{E_{\mathrm{B}-\mathrm{V}}}$.

For a summary of definitions and quantities related to extinction, see, for instance, Whittet (2003). Our designations $E_{\lambda}$ and $T_{\lambda}$ are not standard, but short; the common nomenclature has lengthy expressions like $\left(A_{\lambda}-A_{\mathrm{V}}\right) / E_{\mathrm{B}-\mathrm{V}}$ instead of $E_{\lambda}$. The dust albedo will be denoted by

$\omega=\frac{\tau^{\mathrm{sca}}}{\tau}=\frac{K^{\mathrm{sca}}}{K^{\mathrm{ext}}}$.

We will use the term "aperture" to indicate the area on the sky from which photons are collected in the photometric measurement of a star. In modern pixel array detectors, the "aperture" is determined by the point spread function, in older ones by the diaphragm.

\section{Effective extinction}

\subsection{The extinction optical depth $\tau$}

Photons on their direct way from the star to the telescope are removed by absorption or by scattering. One usually detects only photons that did not interact with the dust. Photons not initially heading towards the observer may also fall into the telescope after having been scattered, but in the standard scenario of stellar photometry their number is negligible. To see why, consider a cloud of low extinction optical thickness $(\tau \ll 1)$ located halfway between the star (distance $D$, luminosity $L$ ) and the Sun. Via absorption and scattering exactly along the line of sight, the cloud reduces the stellar flux, $F=L / 4 \pi D^{2}$, by an amount

$\Delta F=L \tau / 4 \pi D^{2}$.

Let $\Omega$ be the solid angle of the aperture. As the star and the observer are equidistant from the cloud, the fraction of stellar light that is (isotropically) scattered within the aperture equals $\tau^{\text {sca }} \Omega / 4 \pi$. At the position of the observer, it yields a flux

$F^{\mathrm{sca}}=\frac{L \tau^{\mathrm{sca}} \Omega}{4 \pi \cdot 4 \pi\left(\frac{D}{2}\right)^{2}}=\omega \frac{\Omega}{\pi} \Delta F \ll \Delta F$.

For an albedo $\omega \simeq 0.5$ and an aperture $\Omega \simeq 2 \operatorname{arcsec}^{2}$, one finds $\omega \Omega / \pi \sim 10^{-11}$. The scattered light that reaches the telescope is therefore irrelevant and the weakening of the flux by dust is adequately described by $\mathrm{e}^{-\tau}$.

\subsection{The effective optical depth $\tau^{\text {eff }}$}

The situation can be quite different when the star is in another galaxy. A resolution of 1 arcsec translates in the case of the nearest galaxies (Magellanic Clouds) into a linear scale of $0.3 \mathrm{pc}$. To detect a distant star, it must be luminous and it is then usually young and associated with a nebulosity and a molecular cloud complex. The visual optical depth within an aperture $0.3 \mathrm{pc}$

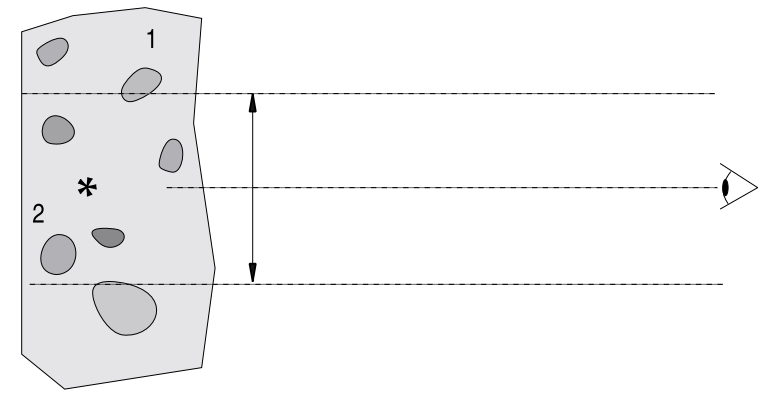

Fig. 1. Observing a star in a clumpy medium. The size of the aperture at the distance of the medium is indicated by the arrow.

across is about 0.5 is the gas density is $1000 \mathrm{~cm}^{-3}$ (assuming standard dust). In such a case, as we will show below, the detected scattered light must be included when dereddening the star.

The scattered light is also important in extragalactic observations at short wavelength $(\lambda \lesssim 3 \mu \mathrm{m})$ when individual stars are not resolved. The target may be a section of a spiral arm, an HII region compex or a central starburst. The radiative transfer is then more complicated than for single stars.

Let $F_{\lambda}$ be the flux that one receives from a star (or several stars) where there is dust in the aperture, and $F_{\lambda}^{\text {nd }}$ the flux that one would receive if there were none (no dust). In the standard scenario, the spatial resolution is small and the dust resides in a cloud well detached from the star. One then has, as shown above, the almost perfect approximation

$F_{\lambda}=F_{\lambda}^{\mathrm{nd}} \mathrm{e}^{-\tau_{\lambda}}$.

In the dust distribution sketched in Fig. 1, however, the detected scattered photons must not be neglected. We define for this general case an effective optical thickness, $\tau_{\lambda}^{\text {eff }}$, by

$F_{\lambda}=F_{\lambda}^{\mathrm{nd}} \mathrm{e}^{-\tau_{\lambda}^{\mathrm{eff}}}$

Instead of $\tau^{\text {eff }}$, one also finds in the literature the terms $\tau^{\mathrm{cs}}$ ( $\mathrm{cs}=$ circumstellar $), \tau^{\mathrm{sc}}(\mathrm{sc}=$ scatter $)$ and $\tau^{\mathrm{att}}$ (att $=$ attenuation $)$. The effective extinction curve is given by

$T_{\lambda}^{\mathrm{eff}}=\frac{\tau_{\lambda}^{\mathrm{eff}}}{\tau_{\mathrm{V}}^{\mathrm{eff}}}$

or by

$E_{\lambda}^{\mathrm{eff}}=\frac{\tau_{\lambda}^{\mathrm{eff}}-\tau_{\mathrm{V}}^{\mathrm{eff}}}{\tau_{\mathrm{B}}^{\mathrm{eff}}-\tau_{\mathrm{V}}^{\mathrm{eff}}}$

Like $E_{\lambda}$, it has to be determined by comparing the fluxes of the star with dust in the aperture with those of a star of the same spectral shape without dust. In analogy to $R_{\mathrm{V}}$, one can define

$R_{\mathrm{V}}^{\mathrm{eff}}=\frac{\tau_{\mathrm{V}}^{\mathrm{eff}}}{\tau_{\mathrm{B}}^{\mathrm{eff}}-\tau_{\mathrm{V}}^{\mathrm{eff}}}$.

The effective optical thickness $\tau^{\text {eff }}$ refers, like $\tau$, to a specific source, but, unlike $\tau$, also to a particular observational set-up (aperture). The effective extinction curve $E_{\lambda}^{\text {eff }}$ depends on the source and the set-up, whereas $E_{\lambda}$ is a fixed function.

Because of this lack of generality one may wonder why one should bother with $\tau^{\text {eff }}$ and $E_{\lambda}^{\text {eff }}$ at all. First, observations can only yield $E_{\lambda}^{\text {eff }}$. Second, sometimes $E_{\lambda}^{\text {eff }}$ and $E_{\lambda}$ differ and then apparent anomalies have to be blamed on the reflected light, and not on the source or the dust properties. Third, one needs $\tau_{\lambda}^{\text {eff }}$ to deredden a source and to determine its intrinsic spectral luminosity. 


\subsubsection{How to compute $\tau^{\text {eff }}$}

A general procedure to obtain $\tau^{\text {eff }}$, which we will employ in the Monte Carlo computations (Sect. 4.2), is the following. Suppose the star emits $Z^{*}$ photons (we drop the subscript $\lambda$, unless it is really necessary). If the telescope subtends, as seen from the star, the solid angle $\theta_{\text {tel }}$, an observer would detect $Z^{\text {nd }}=Z^{*} \theta_{\text {tel }} / 4 \pi$ photons if there were no dust. In the presence of dust, the observer sees

$Z^{\text {nd }} \mathrm{e}^{-\tau^{\text {eff }}}=Z^{*} \frac{\theta_{\text {tel }}}{4 \pi} \mathrm{e}^{-\tau}+Z^{\text {sca }}$

photons, $Z^{\text {dir }}=Z^{*} \mathrm{e}^{-\tau} \theta_{\text {tel }} / 4 \pi$ of which come towards him directly, on a straight line without interaction in the cloud, and $Z^{\text {sca }}$ reach him after having been scattered once or repeatedly. The locus of the last scattering event must lie within the aperture (for example, point 2 in Fig. 1, but not point 1). From Eq. (9) follows

$\tau^{\mathrm{eff}}=\tau-\ln \left[1+\frac{Z^{\mathrm{sca}}}{Z^{*}} \frac{4 \pi}{\theta_{\mathrm{tel}}} \mathrm{e}^{\tau}\right]$.

Therefore, the effective optical thickness

- is always smaller than or equal to $\tau$;

- can be negative, i.e. more light is detected when there is dust than when there is none;

- approaches $\tau$ when the aperture is small because $Z^{\text {sca }}$ tends to zero. In this case, $E_{\lambda}^{\text {eff }}$ coincides with the standard extinction curve $E_{\lambda}$.

Equations (9) and (10) apply to one star. When the aperture contains a dusty star cluster, its effective optical depth is obviously given by

$\mathrm{e}^{-\tau^{\mathrm{eff}}}=\sum_{i} f_{i} \mathrm{e}^{-\tau_{i}^{\mathrm{eff}}}$

where $\tau_{i}^{\text {eff }}$ is the effective extinction of star $i$ and $f_{i}$ its fractional luminosity with $\sum f_{i}=1$. The $\tau_{i}^{\text {eff }}$ can be evaluated for each star independently of the others.

- Suppose there are $N$ stars in the cluster. If all are identical, $\mathrm{e}^{-\tau^{\text {eff }}}=N^{-1} \sum \mathrm{e}^{-\tau_{i}^{\text {eff }}}$. If they are furthermore located in such a way that $\tau_{i}^{\text {eff }}=$ const (for instance, as in Figs. 4 and 5 where even $\tau_{i}=$ const), then $\tau^{\text {eff }}=\tau_{i}^{\text {eff }}$.

- When the cluster is transparent (all $\tau_{i}<1$ ), Eq. (11) yields $\tau^{\mathrm{eff}}=\sum f_{i} \tau_{i}$. The effective optical depth is then determined by those stars for which the product $f_{i} \tau_{i}$ is highest.

- When the $\tau_{i}$ are not small, the correction factor $\mathrm{e}^{-\tau^{\mathrm{eff}}}$ in Eq. (5) is determined by the stars for which $f_{i} \mathrm{e}^{-\tau_{i}^{\text {eff }}}$ is greatest. This means that the visible cluster luminosity, and its color, does not necessarily come from the brightest stars, but from those that suffer the least attenuation; a rather self-evident, but important result.

\section{The radiative transfer}

\subsection{Geometry and method}

Dust and stars can be arranged in an endless variety. The geometries may be classified according to whether

- the medium is homogeneous or clumpy;

- one is dealing with one, several or many stars;

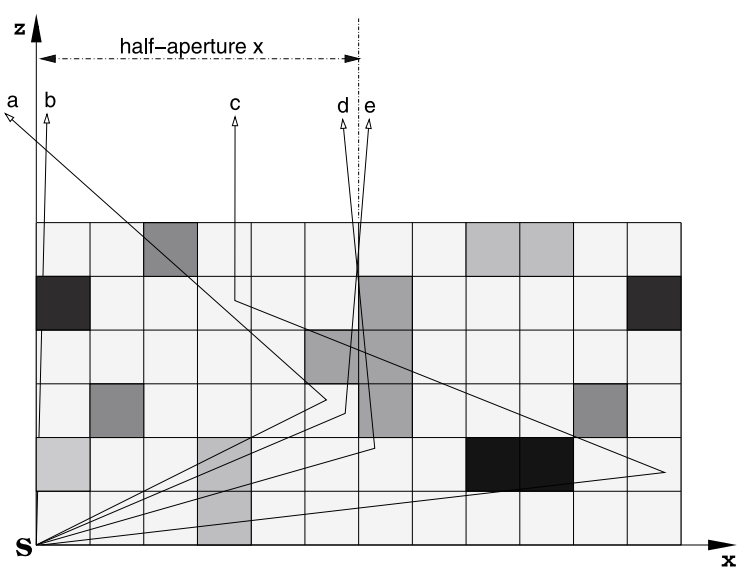

Fig. 2. A 2d-illustration of the first octant of a clumpy cloud showing five photon tracks. The $z$-axis points towards the observer who is located at $(x, y, z)=(0,0, \infty)$. Here only one star is at $S$, but is some tasks, there are many stars.

- the structure is multi-dimensional or depends on only one coordinate, for instance, the $z$-axis of an infinite slab;

- $\tau^{\text {eff }}$ depends on the aperture, as in Fig. 1, or is independent of it, as in Figs. 4 or 5.

We study the attenuation of light for six idealized, but basic configurations when the dust medium is homogeneous (sphere, infinite slab, block). We vary in the computations the extinction optical depth, the albedo, the phase function, the aperture (where it makes sense) and the source distribution (one point source or many stars continuously smeared out). Although the number of basic geometries is not large, our study is far from exhaustive.

We also investigate in two cases (infinite slab and block) the extinction by a clumpy medium. Besides tremendously enlarging the parameter space, an element of randomness enters the results through the selection of a concrete geometry.

The radiative transfer is always relatively simple because dust emission can be disregarded. We employ various techniques to compute the effective optical depth $\tau^{\text {eff }}$ which we now describe briefly.

\subsection{Radiative transfer with Monte Carlo}

We employ a Monte Carlo technique whenever

- the medium is clumpy or;

- the geometry does not possess 1d-symmetry (for instance, when it is shaped like a brick) or;

- scattering is anisotropic.

\subsubsection{The space grid in $\mathrm{MC}$}

We use Cartesian coordinates and consider a block built up of cubes as illustrated in Fig. 2 in two dimensions (where the cubes have become squares). Only the octant with $x \geq 0, y \geq 0, z \geq 0$ is shown. There are typically $\sim 10^{7}$ cubes per octant, but in some tasks up to $2 \times 10^{8}$. The density is constant within a cube, but it may change from one cube to the next.

To reduce the computer demand, we always assume mirror symmetry with respect to the planes $x=0$ and $y=0$, but not necessarily with respect to the plane $z=0$.

When a photon travelling in the direction of the unit vector $\left(e_{x}, e_{y}, e_{z}\right)$ with $e_{x}<0$ crosses the plane $x=0$ at some position $\boldsymbol{r}$ - this can only happen after scattering - and thus leaves the first 
octant in which the calculations are performed and enters the neighboring octant, it is, because of mirror symmetry, replaced by a photon entering the first octant at $\boldsymbol{r}$ in the direction $\left(-e_{x}, e_{y}\right.$, $e_{z}$ ); likewise for the plane $y=0$.

When not interacting with the dust, a photon traverses a cube on a straight line. If $\Delta \tau$ is the extinction optical depth between the entry and exit point, both on the cube surface, and if $r \in[0,1]$ is a random number, the photon interacts with the dust, i.e. it is absorbed or scattered within the cube if

$\Delta \tau \geq-\log (r)$

The probability for scattering is equal to the albedo $\omega$, and for absorption is $1-\omega$. An absorbed photon is lost. After scattering, the photon changes direction. The new direction is in a probabilistic way determined by the phase function.

We consider below four configurations of stars and dust. In the first two, there is only one star at position $(x, y, z)=(0,0,0)$ (denoted $S$ in Fig. 2), in the last two, we model an infinite slab. A sufficient approximation to infinity is a block that is flat and several times broader than the aperture. If Fig. 11 delineates the dimensions of such a block, $L / h \gtrsim 10$ and $L / a \gtrsim 5$. The extinction optical thickness in the $(x, y)$-plane must be high enough $(\tau \gtrsim 10)$ so that photons from the edge of the slab have almost no chance of reaching the aperture. When modeling a clumpy medium, the aperture should be larger than the largest turbulent scale.

The four configurations are:

1. there is one star at $S$, the space for $z<0$ is empty;

2 . there is one star at $S$, which is the cloud center. The cloud is mirror-symmetric with regard to the plane $z=0$; the space with $z<0$ is not empty;

3. there are many identical stars evenly distributed in the $(x, y)$-plane of the "infinite" slab; in each square of the grid there is one star (Fig. 2);

4. there are many identical stars evenly distributed all over the "infinite" slab; there is one star in each grid cube.

\subsubsection{The solid angle of the telescope mirror}

In Eq. (10), $\theta_{\text {tel }}$ is the solid angle under which the telescope mirror is viewed from the star. Because it is much too small for any practical computation, we replace the true mirror by a fictitious giant mirror with an angular diameter, as seen from the source, of one or a few degrees. The calculation will still be basically correct as long as the appearance of the source changes only mildly if it were rotated by a demon by one or a few degrees. This should be true for most sources. For instance, we do not expect the Orion nebula and the stars within it to look very different after rotation by such a relatively small amount.

However, if there is strong small-scale clumping in the source, the calculation applies only to the photometry with the fictitous giant mirror, or to the average of many measurements made with normal-sized telescopes uniformly spread over the area of the giant mirror. The appearance of the source may vary drastically from one telescope to another at a slightly different position while $\tau$ may change value from zero to huge. On the other hand, the effective extinction curve for two positions with very discrepant $\tau$ need not differ much. Exactly how much has to be derived from a MC computation.

In the case of a giant mirror, four of the tracks shown in Fig. 2 ( $b, c, d$ and $f$ ) will end at the telescope (the angle with the $z$-axis is of the order 1 degree) while track $a$ (where the angle with the

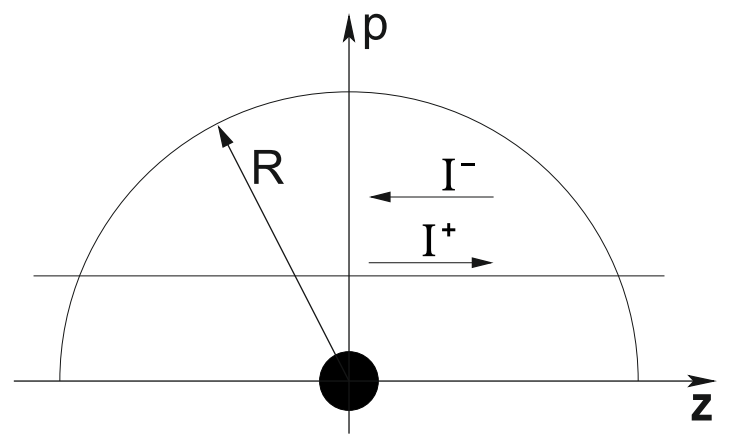

Fig. 3. Ray tracing in a sphere of radius $R$ on lines $p=$ const from left to right $\left(I^{+}\right)$and back $\left(I^{-}\right)$.

$z$-axis is large) and track $e$ (the scattered photon originates from a point outside the aperture) will pass by.

\subsection{Ray tracing}

In ray tracing, one solves the transfer equation (omitting the frequency index)

$\frac{\mathrm{d} I}{\mathrm{~d} \tau}=-I+S$

in all directions together with the boundary conditions. When the medium is densely packed with stars and $\epsilon$ denotes the emissivity (i.e. the energy emitted by the stars per unit time, volume, solid angle and frequency interval), then, without dust emission, the source function in the direction $\boldsymbol{e}$ is given by

$S(\boldsymbol{e})=\frac{\epsilon}{K^{\mathrm{ext}}}+\frac{\omega}{4 \pi} \int p\left(\boldsymbol{e}^{\prime}, \boldsymbol{e}\right) I\left(\boldsymbol{e}^{\prime}\right) \mathrm{d} \Omega^{\prime}$.

Here $p\left(\boldsymbol{e}^{\prime}, \boldsymbol{e}\right)$ is the probability of scattering from direction $\boldsymbol{e}^{\prime}$ into direction $\boldsymbol{e}$. Without stars, $\epsilon=0$.

For isotropic scattering, $p=1$ and $S=\omega J$ where $J$ denotes the mean intensity. The numerical solution of Eq. (13) then requires an integration over $\tau$ and one over all directions of the intensity $I$. When scattering is anisotropic, the source function also depends on direction and one has to perform at each spatial grid point another double integration over the solid angle $4 \pi$. We therefore solve the radiative transfer in the case of anisotropic scattering with Monte Carlo.

\subsubsection{Sphere with a central star}

We solve Eq. (13) for the intensity in positive and negative directions, $I^{+}$and $I^{-}$, along lines of constant impact parameter $p$ as shown in Fig. 3. The star has a radius $R_{*}$ and is at the cloud center. If its luminosity $L=4 \pi R_{*}^{2} F_{*}$, the inner boundary condition reads $I^{+}-I^{-}=F_{*} / \pi$ on the stellar surface and $I^{+}=I^{-}$when $z=0$ and $p>R_{*}$. On the cloud surface, $I^{-}=0$.

\subsubsection{Uniform, infinite slabs}

A homogeneous plane screen, infinite in the $(x, y)$-plane and of extinction optical depth $\tau_{0}$ along the $z$-axis, is illuminated by a source, smoothly distributed and also unbounded in the $x$ - and $y$-direction; it may lie either inside or behind the screen. The basic transfer equation reads

$\mu \frac{\mathrm{d} I}{\mathrm{~d} \tau}=-I+S$ 


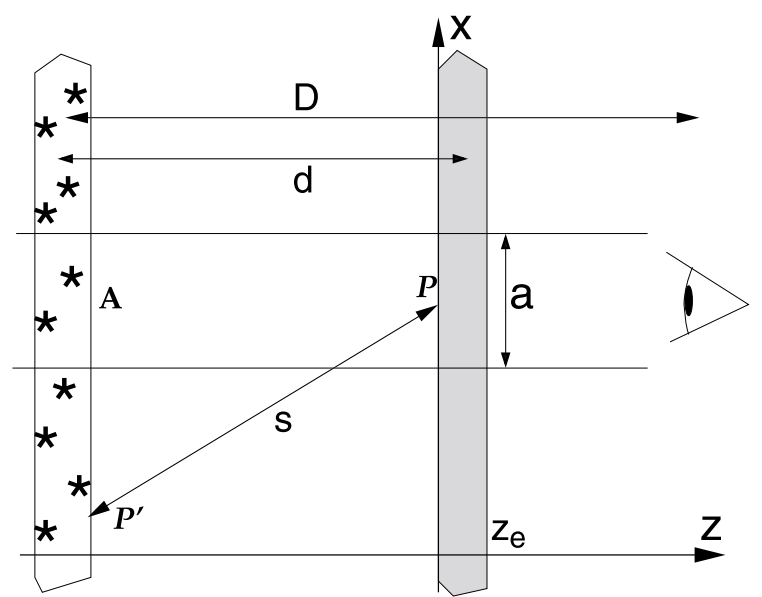

Fig. 4. An infinite slab uniformly filled with stars is behind an infinite homogeneous dust screen that extends from $z=0$ to $z=z_{e}$. The distance $D$ from point $A$ to the observer, the spacing $d$ between slab and screen as well as the linear size, $a$, of the aperture on the screen are auxiliary quantities used only in appendix A.

$\mu=\cos \theta$ is the angle between the direction of the light ray and the $z$-axis and $\tau$ is measured along $z$. An observer, looking perpendicularly onto the screen, detects the intensity $I(\tau=$ $\left.\tau_{0}, \mu=1\right)$ and, if $\Omega$ is the solid angle of the aperture, the flux $F=\Omega I\left(\tau_{0}, 1\right)$.

If the screen were removed (no dust), the detected intensity would be $I^{\text {nd }}$ and the flux $F^{\text {nd }}=\Omega I^{\text {nd }}$. Therefore, according to Eq. (5), $I=I^{\text {nd }} \mathrm{e}^{-\tau^{\text {eff }}}$. To find the effective optical thickness, one has to compute $I\left(\tau_{0}, 1\right)$. We discuss three configurations.

a) Stellar slab behind a dust screen: the screen is illuminated from behind by a plane-parallel infinite slab that is evenly filled with point-like stars. Their space density is high so that looking in Fig. 4 from $P$ towards the slab, the intensity, even when averaged over a small solid angle, changes smoothly from one place (say, point $P^{\prime}$ ) to another.

Let $I^{+}$be the intensity of light traveling to the right and $I^{-}$ of light going to the left. If $I_{0}$ is the average intensity from $P$ towards a small area around $A$ in Fig. 4 , the boundary conditions to Eq. (14) are

$I^{-}\left(z=z_{e}, \mu\right)=0, \quad I^{+}(z=0, \mu)=\frac{I_{0}}{\mu}$.

b) Stars in the midplane of a dust screen: the point-like stars are evenly and densely packed at $z=0$ (Fig. 5); this is now the symmetry plane. The screen extends from $-z_{\mathrm{e}}$ to $z_{\mathrm{e}}$. The component of the flux in the $z$-direction, $F_{z}$, has a discontinuity at $z=0$ changing from $-2 \pi I_{0}$ for $z<0$ to $+2 \pi I_{0}$ at $z>0$. If $h$ is an infinitesimaly small elevation above the midplane, the boundary conditions read

$$
\begin{aligned}
I^{-}\left(z=z_{\mathrm{e}}, \mu\right) & =0 \\
I^{+}(h, \mu)-I^{-}(h, \mu) & =\frac{I_{0}}{\mu} .
\end{aligned}
$$

Again, $I_{0}$ is the average intensity which the observer would see looking towards a small area around point $A$ in Fig. 5 if there were no dust. The intensity $I^{+}(h, \mu)$ also now has a contribution from light that has been backscattered in the half-space with $z<0$.

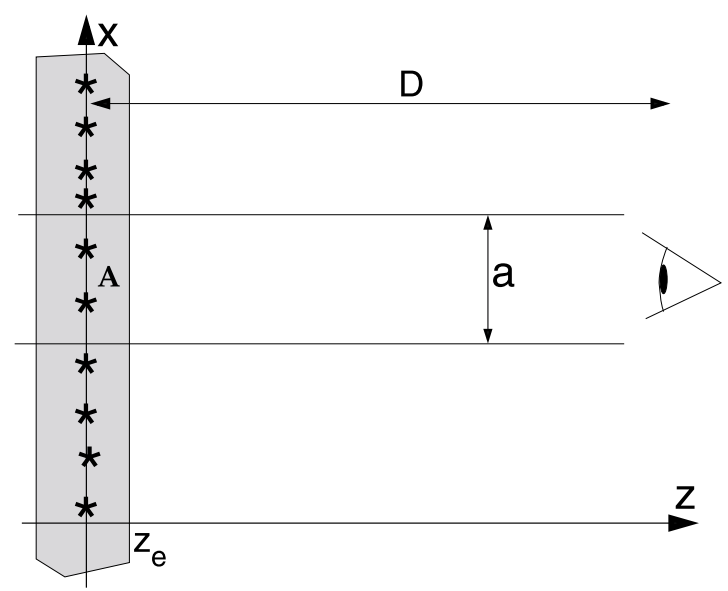

Fig. 5. Stars in the midplane of a homogeneous, infinite dust screen; $a$ is the linear size of the aperture.

c) Uniform slab of stars and dust: the slab extends from 0 to $z_{e}$ and is evenly filled with stars; the emissivity $\epsilon$ is constant. The boundary conditions now read

$I^{-}\left(z_{\mathrm{e}}, \mu\right)=0, \quad I^{+}(0, \mu)=0$.

When scattering is isotropic and $J$ is the mean intensity, the source function becomes

$S=\frac{\epsilon}{K^{\mathrm{ext}}}+\omega J$

For $\mu=1$ and in the case of pure absorption $\left(\omega=0, K^{\text {ext }}=\right.$ $K^{\text {abs }}$ ), Eq. (14) has the solution

$I^{+}(\tau)=\left(1-\mathrm{e}^{-\tau}\right) \frac{\epsilon}{K^{\mathrm{ext}}}=\frac{1-\mathrm{e}^{-\tau}}{\tau} \epsilon z$.

It yields $I^{+}(0)=0$ and $I^{+}\left(\tau_{e}\right)=\left(1-\mathrm{e}^{-\tau_{e}}\right) \epsilon z_{e} / \tau_{e}$ where $\tau_{e}=$ $z_{e} K^{\text {ext }}$. Without dust, $I^{+}\left(z_{e}\right)=z_{e} \epsilon$ and therefore (cf. Eq. (29))

$\mathrm{e}^{-\tau^{\mathrm{eff}}}=\frac{1-\mathrm{e}^{-\tau}}{\tau}$

\section{Optical dust properties}

When calculating the effective extinction curve, we assume standard dust which means, first of all, that the normalized extinction coefficient, $K_{\lambda}^{\text {ext }} / K_{\mathrm{V}}^{\text {ext }}$, exactly matches the standard reddening curve $T_{\lambda}$ for which $R_{\mathrm{V}}=3.1$. The absolute value of $K_{\lambda}^{\text {ext }}$ (in $\mathrm{cm}^{2}$ per gram of dust) is not needed. We also have to specify the albedo, $\omega$, and the phase function $f(\cos \theta)$, where $\theta$ denotes the scattering angle. For the latter, we adopt (Henyey \& Greenstein 1941)

$f_{\lambda}(\cos \theta)=\frac{1-g_{\lambda}^{2}}{\left(1+g_{\lambda}^{2}-2 g_{\lambda} \cos \theta\right)^{3 / 2}}$.

The asymmetry factor, $g$, is related to $f(\cos \theta)$ by

$g=\frac{1}{2} \int_{-1}^{1} x f(x) \mathrm{d} x$

For isotropic scattering, $g=0$. In pure forward scattering which is equivalent to no scattering at all, $g=1$.

The albedo $\omega_{\lambda}$ is observationally not well determined, and the phase function $f_{\lambda}(\cos \theta)$ even less so. As educated guesses, we use for $\omega_{\lambda}$ and $g_{\lambda}$ the values displayed in Fig. 6. They come 


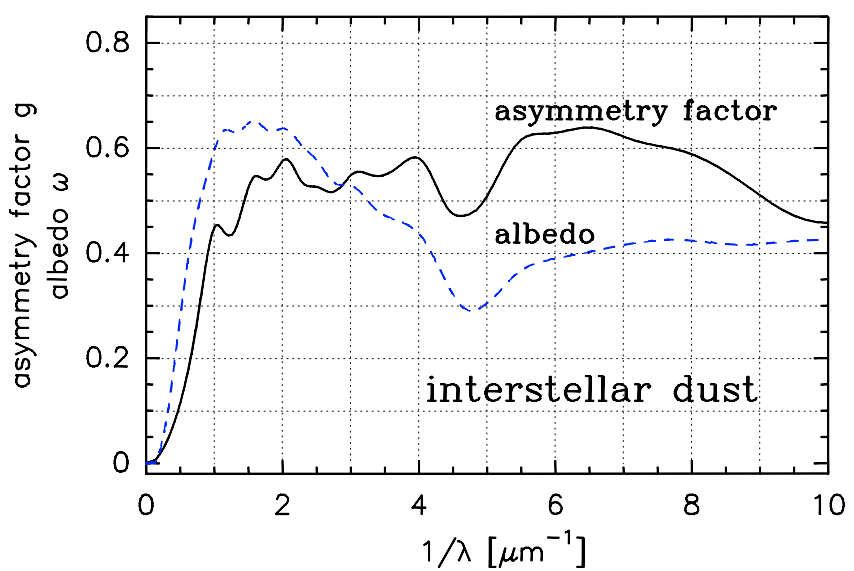

Fig. 6. Albedo $\omega$ and asymmetry factor $g$ of interstellar dust (adapted from Fig. 10.5 of Krügel 2006).

from a simple dust model that meets all major observational constraints (details in Sect. 10.2.2 of Krügel 2006). Among others, it fits the extinction curve $E_{\lambda}$ or $T_{\lambda}$ well, although not perfectly, especially in the far UV, but the small mismatches are irrelevant. We point out that Eq. (21) is not strictly consistent with the dust model itself which is based on Mie theory and yields a different form of $f_{\lambda}(\cos \theta)$, but fortunately, for a given $g_{\lambda}$, the radiative transfer results depend little on the phase function.

The albedo and the asymmetry factor of the model are, within the uncertainties, in agreement with what one deduces from observations (see, for instance, Figs. 1 to 4 in Gordon 2004). We summarize their behavior. a) In the infrared, $\omega_{\lambda}$ and $g_{\lambda}$ are both small. For $\lambda \gtrsim 2 \mu \mathrm{m}$, the albedo stays below 0.25 and $g_{\lambda} \simeq \omega_{\lambda}$ so that isotropic scattering is not a bad approximation; b) the albedo peaks at optical wavelengths $\left(\omega_{\mathrm{V}} \simeq 0.6\right)$, dips at the $4.6 \mu \mathrm{m}^{-1}$ hump and is constant and around 0.4 in the far UV; c) the asymmetry factor $g_{\lambda}$ behaves similarly. At visual wavelengths, it is large $(g \simeq 0.6)$ and forward scattering dominates.

\section{Effective optical depth vs. extinction optical depth}

To demonstrate the effect of the scattered light, we first compute the effective optical depth, $\tau^{\text {eff }}$, for some of the configurations discussed in Sect. 4 treating the optical dust properties as free parameters. Some of the values for the albedo $\omega$ and asymmetry factor $g$, or their combinations, are even unrealistic and do not apply to "normal" interstellar dust at any wavelength (see Fig. 6).

We will always plot $\tau^{\text {eff }} / \tau$ as a function of the extinction optical depth $\tau$. When $\tau^{\text {eff }} / \tau \simeq 1$, scattered light is negligible and when $\tau^{\text {eff }} / \tau$ is substantially less than one, it is important.

\subsection{A uniform sphere with a central star}

A sphere with a central star applies very well to giants blowing out a dusty wind and roughly to very young stars embedded in the remnants of the protostellar clump. In both cases, the density is centrally peaked. We consider here, however, only homogeneous spheres.

Figure 7 presents results when the whole sphere is contained in the aperture. The extinction optical thickness towards the star varies from $10^{-3}$ to 200 , the albedo has values from zero to one in steps of 0.2 . The solid lines are computed with ray tracing assuming isotropic scattering. For interstellar dust, this assumption

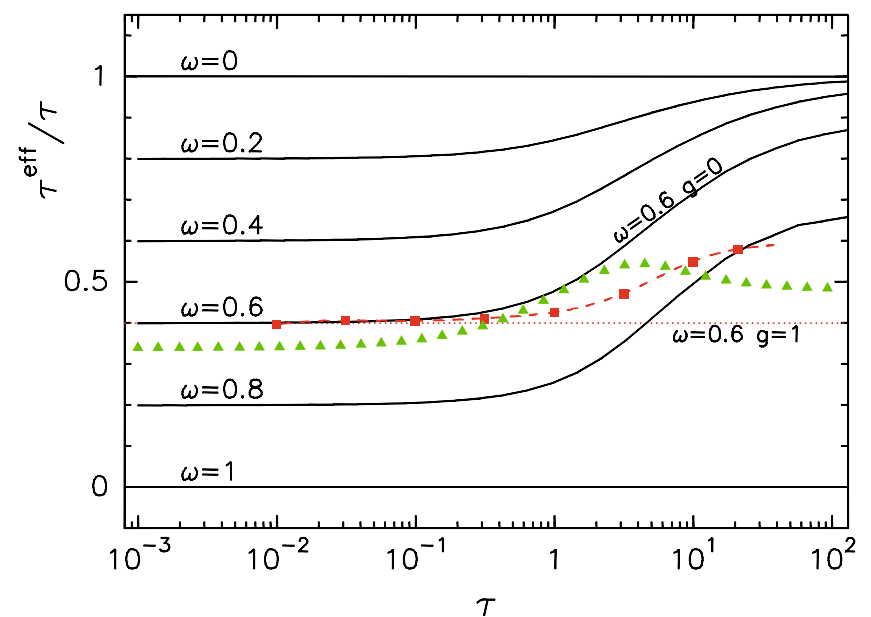

Fig. 7. The ratio of effective optical depth to extinction optical depth, $\tau^{\text {eff }} / \tau$, as a function of $\tau$ for a homogeneous sphere contained in the aperture. Solid lines: isotropic scattering $(g=0)$; dotted line: $(\omega, g)=$ $(0.6,1)$; dashed line: $(\omega, g)=(0.6,0.6)$; triangles: after Eq. $(25)$ from Mathis (1972), also for $(\omega, g)=(0.6,0.6)$.

is approximately correct only in the infrared $(\lambda \gtrsim 2 \mu \mathrm{m})$; at shorter wavelengths, scattering is anisotropic.

As an example, we show the dependence of $\tau^{\text {eff }} / \tau$ on $\tau$ for $(\omega, g)=(0.6,0.6)$. This pair of values is appropriate in the visual. The computations were performed with the Monte Carlo method and therefore do not reach high or low optical depths. The values for $g=0.6$ are bracketed by the solid line $(\omega=0.6, g=0)$ and the dotted one $(\omega=0.6, g=1)$.

The general procedure to approximately determine from Fig. 7 the effective optical depth $\tau^{\text {eff }}$ for any value of $\tau, \omega_{0}$ and $g_{0}>0$ is now the following: a) draw the curve for $\omega_{0}$ and $g=0$, if necessary by interpolating between the solid lines in Fig. 7; b) extend this curve from small $\tau$ parallel to the abscissa to large $\tau$. The resulting curve refers to $\omega_{0}$ and $g=1$; c) interpolate between the curve $g=0$ and $g=1$. It turns out that linear interpolation yields values of $\tau^{\mathrm{eff}} / \tau$ that are accurate to within a few percent.

We summarize salient points for homogeneous spheres.

- It is easy to verify that $\tau^{\mathrm{abs}} \leq \tau^{\mathrm{eff}} \leq \tau^{\mathrm{ext}}$.

- Without scattering, $\omega=0$ and $\tau^{\mathrm{eff}} / \tau=1$.

- When $\omega=1$, no photons are lost and $\tau^{\mathrm{eff}} / \tau=0$.

- When $\tau$ is small or $g$ equals one, $\tau^{\mathrm{eff}} / \tau=1-\omega$ (Eq. A. 1 ) and the effective extinction curve is

$\frac{\tau_{\lambda}^{\mathrm{eff}}}{\tau_{\mathrm{V}}^{\mathrm{eff}}}=\frac{K_{\lambda}^{\mathrm{abs}}}{K_{\mathrm{V}}^{\mathrm{abs}}}$.

- When $\tau$ and $g$ are fixed, $\tau^{\text {eff }} / \tau$ decreases as the albedo increases.

- When $\tau, \omega$ and $g$ are fixed, $\tau^{\text {eff }} / \tau$ grows monotonically with $\tau$.

- For fixed $\omega$, the curve with $g>0$ lies between the one with $g=0$ and $g=1$.

\subsection{Uniform, infinite slabs}

In Sect. 4.3.2 we described the radiative transfer and displayed the underlying geometry for three configurations where a homogeneous infinite dust slab, which we call the screen, lies parallel to an infinite, uniform stellar slab. 


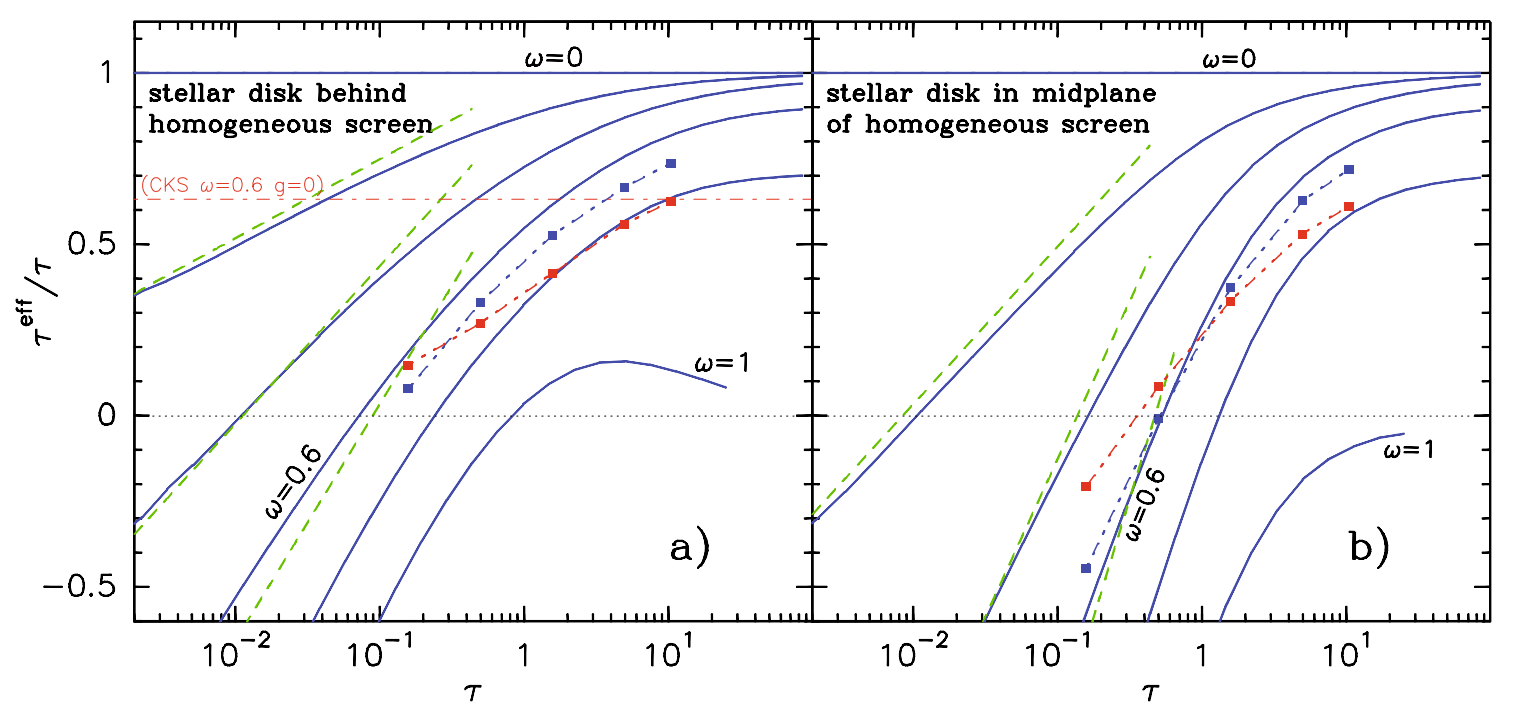

Fig. 8. $\tau^{\text {eff }} / \tau$ as a function of the extinction optical depth $\tau$ for a stellar plane and a homogeneous dust screen. a) The screen is irradiated from behind (Fig. 4); b) the stars are in the midplane of the screen (Fig. 5). Blue solid: isotropic scattering $(g=0)$, the albedo $\omega$ varies from 0 (top curve) to 1 in steps of 0.2. Green dashes: small- $\tau$ approximation of (A.4) and (A.5). Squares: anisotropic scattering for $\omega=0.6$; red: $g=0.6$, blue: $g=0.3$. Red dash-dots: after CKS for $\omega=0.6$ and $g=0$ (see Eq. (27)).

We now discuss how the ratio $\tau^{\text {eff }} / \tau$ depends on $\tau$ and on the albedo. Besides isotropic scattering, we also give examples of anisotropic scattering. As no length scale is defined, the results cannot depend on the aperture nor on the distance between source and screen or the distance from the observer to the source.

\subsubsection{Stellar slab behind a dust screen}

A stellar slab shining through a flat dust screen (Fig. 4) is probably a poor representation of any configuration in the astronomical real world, but as it has been repeatedly treated in the literature (Natta \& Panagia 1984; Caplan \& Deharveng 1986; Calzetti et al. 1994), we also show the results of our computations (Fig. 8a). We point out the following features.

- Without scattering $(\omega=0), \tau^{\mathrm{eff}}=\tau$.

- For isotropic scattering and small $\tau$ (and $\omega<1$ ), Eq. (A.4) gives the approximation $\tau^{\mathrm{eff}} / \tau \simeq 1+\frac{1}{2} \omega \ln \tau^{\mathrm{abs}}$ which fits the radiative transfer calculations reasonably well (dashes in Fig. 8a). When additionally $\omega \rightarrow 1, \tau^{\text {abs }}$ becomes very small, $\ln \tau^{\text {abs }}$ diverges and the agreement worsens.

- For isotropic scattering, $\tau^{\text {eff }}$ becomes negative when $\tau$ is small and the albedo $\omega \gtrsim 0.4$. This means an observer looking perpendicularly onto the stellar slab receives more light when there is dust than when there is none (no screen).

- As expected, when $\tau$ is not too small, forward scattering reduces $\tau^{\mathrm{eff}} / \tau$. As $g \rightarrow 1$ (extreme forward scattering), $\tau^{\mathrm{eff}} \rightarrow(1-\omega) \tau$ (not shown in Fig. 8$)$.

\subsubsection{Stars in the midplane of a dust screen}

When the stars are in the midplane of a dust screen (Fig. 5), the configuration bears some resemblance to the distribution of dust and OB stars in a disk galaxy. In Fig. $8 b, \tau$ is now the vertical optical depth to the star layer, and not through the whole screen.

- As always, $\tau^{\text {eff }}=\tau$ when $\omega=0$.

- For $\tau \ll 1, \tau^{\mathrm{eff}} / \tau \simeq 1+\omega \ln \tau^{\mathrm{abs}}$ (see Eq. (A.5)).

- For $\tau \gg 1$, the light that is scattered at $z<z_{e}$ is negligible and the curves $\tau^{\text {eff }} / \tau$ converge towards those for a star-filled slab in the rear. This can be verified by comparing Figs. 8a and $8 \mathrm{~b}$.

- Again, $\tau^{\text {eff }}$ becomes negative for small $\tau$.

- The effect of anisotropic scattering, even with $g=0.6$, is moderate. At $\tau \sim 1, \tau^{\text {eff }}$ hardly changes relative to $g=0$, at larger $\tau$, it is reduced, at smaller enhanced. As $g \rightarrow 1$, $\tau^{\mathrm{eff}} \rightarrow(1-\omega) \tau$ (not shown).

\subsubsection{A homogeneous slab of stars and dust}

This scenario where stars and dust are evenly mixed is again, at least crudely, representative for a disk galaxy, especially for the population of young stars because their scale height and that of the dust are similar. In Fig. 9, $\tau$ denotes the extinction optical depth perpendicular through the whole slab.

- There is little agreement with the boxes in Fig. 8 where the sources are behind or exactly in the mid-plane; $\tau^{\text {eff }} / \tau$ is now generally much smaller.

- In the case of pure absorption, $\tau^{\mathrm{eff}} / \tau$ no longer equals one, but approaches one half for small $\tau$. This follows also from Eq. (20).

- When $\tau \rightarrow \infty$, the ratio $\tau^{\text {eff }} / \tau$ goes to zero for any $\omega$ because the front layer with $\tau \simeq 1$ is always visible almost without attenuation whereas the stars behind the front layer are weakened exponentially.

- $\tau^{\text {eff }} / \tau$ can again become negative. It happens for $\omega \geq 0.6$, when $\tau<1$.

- Figure 9 also displays some results for anisotropic scattering.

\subsection{A clumpy, infinite dust slab}

To study the effect of clumpiness, we repeat the calculations of Fig. 8a for a turbulent medium. In this scenario, the screen is in front of the stellar layer (Fig. 4). In the MC calculations, the screen is divided into cubes (see Fig. 2); they are radomly filled with dust, some stay empty, but all filled cubes (clumps) are identical, each with an extinction optical depth $\tau_{\mathrm{c}}$. In this way, one obtains a Poisson distribution for the number of clumps 


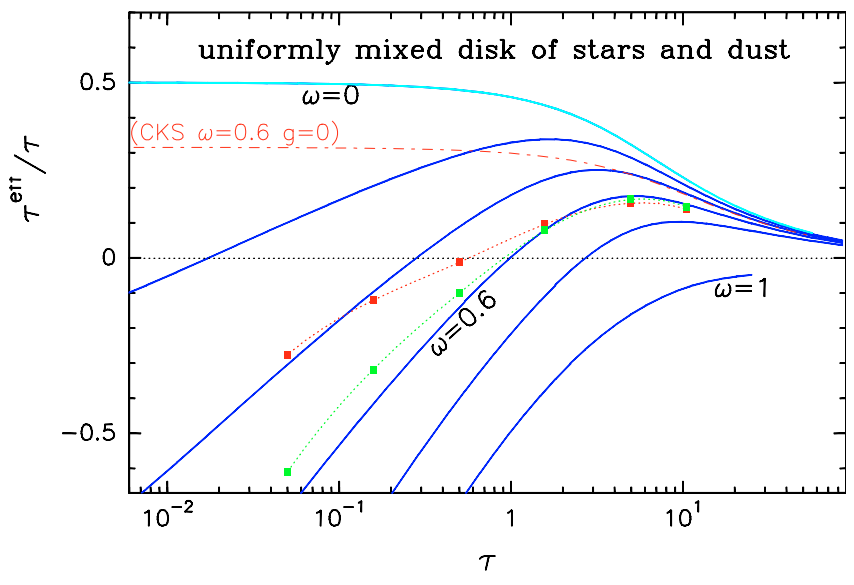

Fig. 9. Stars and dust evenly mixed in an infinite slab. Blue solid: isotropic scattering for $\omega=0$ to 1 in steps of 0.2 ; red dash-dots: after CKS for isotropic scattering and $\omega=0.6$ (see Eq. (29)); squares: anisotropic scattering with $\omega=0.6$, green: $g=0.3$ red: $g=0.6$.

along a line in the $z$-direction. If there are on average $N$ clumps, the mean extinction optical depth is $\tau_{\text {mean }}=N \tau_{\mathrm{c}}$. All stars are in the $(x, y)$-plane where $z=0$ (see Fig. 2). Behind each cube with one face in this plane there is exactly one star.

In Fig. $10, N=3$ and the volume filling factor $f_{\mathrm{Vol}}=0.047$. For isotropic scattering and as long as $\tau_{\text {mean }}$ lies between 0.3 and 2 , the values of $\tau^{\text {eff }}$ in the case of clumpiness (squares) are close to those of a homogeneous screen (solid lines, from Fig. 8a). However, when $\tau_{\text {mean }} \gtrsim 3$, the deviations become large: $\tau^{\mathrm{eff}} / \tau$ is much smaller when the screen is clumped than when it is homogeneous because some light can leak through the screen, between the clumps, without exponential attenuation.

The triangles display one example of anisotropic scattering (albedo $\omega=0.6$, asymmetry factor $g=0.54$ ). The red dashdotted line, which was calculated with $\omega=0.6, g=0$ and $N=3$ is due to CKS (see Eq. (28)) and is clearly an inadequate approximation for a layer of stars behind a turbulent screen.

Figure 10 refers, of course, to a particular parameter set, nevertheless, as was checked in test calculations, it shows the general trend. The excursions from the homogeneous case grow as $N$ and $f_{\mathrm{Vol}}$ decrease while the total dust mass (and $\tau_{\text {mean }}$ ) are kept constant.

\subsection{A homogeneous block with one star}

After infinite slabs, we next consider a single star with a closely associated cloud. Now the dimensions are finite and the aperture comes into play. The cloud is homogeneous and shaped like a brick, or block. It has a height $h$ and a length $L$ in the $x$ - and $y$-directions (Fig. 11). The star is at $(x, y, z)=(0,0,0)$, either in the middle of the block or on its rear face. The aperture is circular and centered on the star and we express its size through

$d=\tan (\Omega / 2)$.

An opening angle $\Omega=28^{\circ}\left(90^{\circ}, 127^{\circ}\right)$ implies $d=0.25(1,2)$. The proportions of the block are determined by the ratio $L / h$. When the star is at the block center, we put in our calculations $L / h=4$, when it is behind, $L / h=8$. These particular choices are not really a restriction, the results stay more or less the same as long as $L \gtrsim 2 a$.

Figure 12 displays the dependence of $\tau^{\mathrm{eff}} / \tau$ on $d=\tan (\Omega / 2)$. Scattering is isotropic $(g=0)$, except in box $c)$ where it is forward, with $g=0.6$.

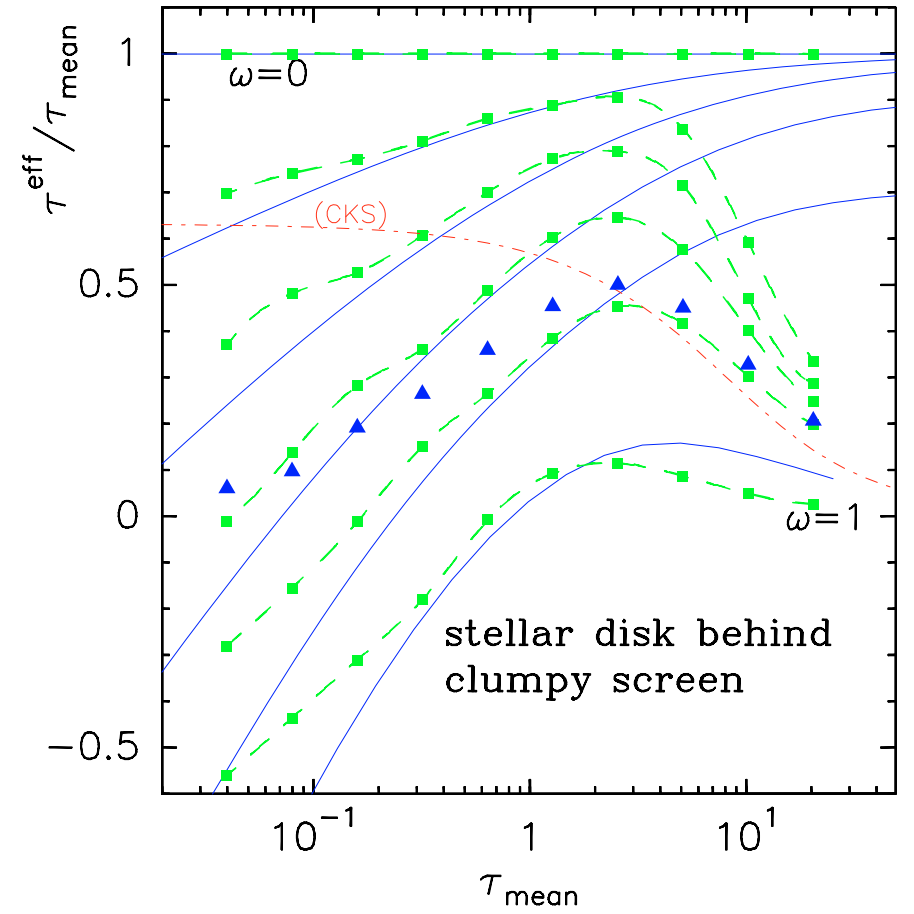

Fig. 10. Clumpy dust screen screen in front of stellar disk. Green squares: isotropic scattering $(g=0), \omega$ from zero to one in steps of 0.2 . Triangles: anisotropic scattering with $\omega=0.6$ and $g=0.54$. Red dashdots: after CKS for $g=0$ and $\omega=0.6$. Solid blue: Homogeneous screen, $g=0$; these curves are from Fig. $8 \mathrm{a}$ and serve as benchmarks.

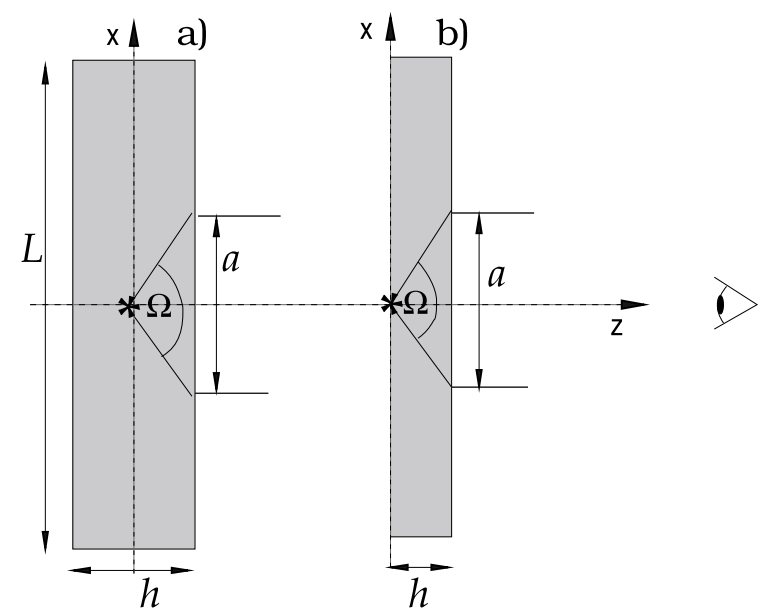

Fig. 11. A homogeneous block with a star in its center (left) or on its rear side $($ right $)$. The aperture defines at the distance of the cloud a length $a$. Its size can also be expressed by the angle $\Omega$ or by $d=\tan (\Omega / 2)$.

- Whenever $\omega=0$ or $g=1, \tau^{\text {eff }}=\tau$.

- For $d \rightarrow 0$ (pencil beam), $\tau^{\text {eff }} / \tau \rightarrow 1$.

- As the angle $\Omega$ widens, the observed flux goes up (as long as $a \leq L)$ and $\tau^{\text {eff }} / \tau$ goes down. The curves become flat at large $d$.

- When $\tau, \omega, d$ and $g$ are fixed, $\tau^{\text {eff }} / \tau$ is always smaller when the star is in the center than when it is behind the cloud.

- When the albedo and the aperture are large and the extinction optical depth small, $\tau^{\text {eff }}$ is negative.

- Forward scattering enhances $\tau^{\text {eff }}$ relative to isotropic scattering when $\tau<1$ and decreases it when $\tau>1$ (see panel c). 


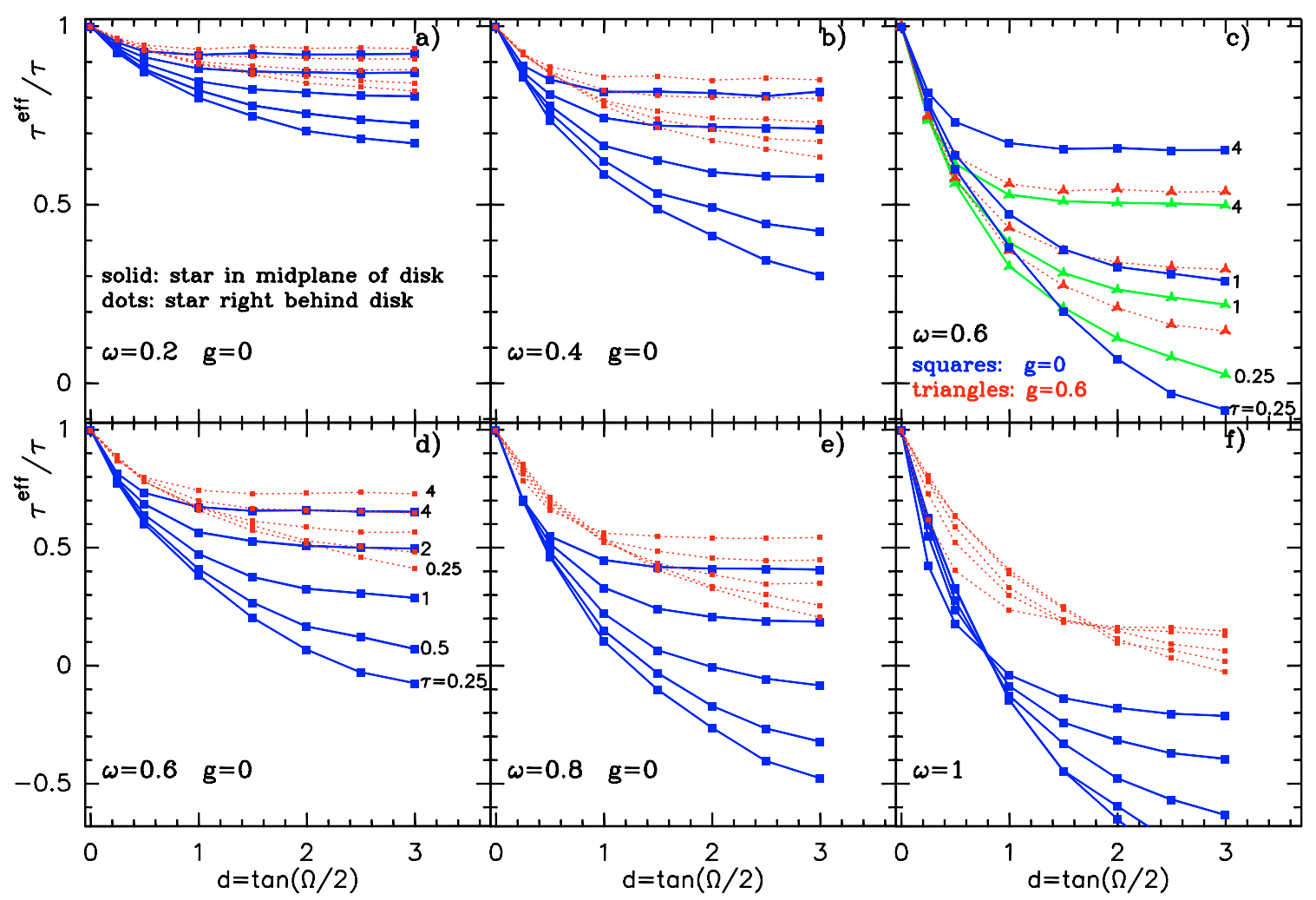

Fig. 12. $\tau^{\text {eff }} / \tau$ vs. the $d=\tan \Omega / 2$ which is a measure of the aperture size (see Fig. 11). The extinction optical depth has the values $\tau=\frac{1}{4}$ (bottom curve), $\frac{1}{2}, 1,2$ and 4 (top curve). The star is either in the midplane of the slab (blue solid lines) or immediately behind it (red dots). Scattering is isotropic $(g=0)$, except in box $c$ ). There the triangles refer to forward scattering with $g=0.6$; the curves with $g=0$ (squares) are taken from box $d$ ) and drawn for comparison. In box c), $\tau$ has only the values $\frac{1}{4}, 1$ and 4 .

- $\tau^{\mathrm{eff}} / \tau$ rises monotonically with $\tau$ when all other parameters are fixed.

\section{The effective extinction curve}

\subsection{Qualitative estimates}

Before presenting computed effective extinction curves, we first estimate, using Figs. 7 to 10 and 12, how we expect $T_{\lambda}^{\text {eff }}$ to differ from the standard reddening curve; predictions for $E_{\lambda}^{\text {eff }}$ are much harder, although one follows from the other. Consider, for example, a single star in a homogeneous block with "standard" dust where we think we know the optical properties $\left(\omega_{\lambda}, g_{\lambda}\right.$ and $\left.K_{\lambda}^{\mathrm{ext}} / K_{\mathrm{V}}^{\mathrm{ext}}\right)$.

- For $\lambda<0.55 \mu \mathrm{m}$, both $g_{\lambda}$ and $\omega_{\lambda}$ are roughly constant (Fig. 6). Because at these wavelengths $\tau_{\lambda}>\tau_{\mathrm{V}}$, we conclude from Fig. 12 that $\tau_{\lambda}^{\text {eff }} / \tau_{\lambda}>\tau_{\mathrm{V}}^{\text {eff }} / \tau_{\mathrm{V}}$, quite independent of the size of the aperture, and therefore (see Eqs. (1) and (6))

$T_{\lambda}^{\mathrm{eff}}>T_{\lambda}$.

- For $0.55 \mu \mathrm{m}<\lambda<0.9 \mu \mathrm{m}, g_{\lambda}$ and $\omega_{\lambda}$ are still roughly constant, but now $\tau_{\lambda}<\tau_{\mathrm{V}}$, therefore

$T_{\lambda}^{\mathrm{eff}}<T_{\lambda}$.

- For $\lambda>0.9 \mu \mathrm{m}$, the situation is unclear and a proper calculation is necessary (now $\tau_{\lambda}<\tau_{\mathrm{V}}$ and $\omega_{\lambda}<\omega_{\mathrm{V}}$, which affects $\tau_{\lambda}^{\text {eff }} / \tau_{\mathrm{V}}^{\text {eff }}$ in opposite ways).

- In the infrared, the albedo drops quickly to zero: $\omega_{\mathrm{J}} \simeq$ $0.38, \omega_{\mathrm{K}} \simeq 0.17$ and $\omega_{\mathrm{M}} \simeq 0.025$. Because $\tau_{\lambda}^{\mathrm{eff}} / \tau_{\lambda} \rightarrow 1$ as $\omega_{\lambda} \rightarrow 0$, one expects at long wavelengths $1 \simeq \tau_{\lambda}^{\mathrm{eff}} / \tau_{\lambda}>$ $\tau_{\mathrm{J}}^{\mathrm{eff}} / \tau_{\mathrm{J}}$ which implies

$\frac{\tau_{\lambda}^{\mathrm{eff}}}{\tau_{\mathrm{J}}^{\mathrm{eff}}}>\frac{\tau_{\lambda}}{\tau_{\mathrm{J}}}$.

The effective exctinction curve should therefore lie above the standard curve for $\lambda<0.55 \mu \mathrm{m}$ and below it for $0.55 \mu \mathrm{m}<\lambda \leq$ $0.9 \mu \mathrm{m}$. When normalized at $J$, one expects $\tau_{\lambda}^{\mathrm{eff}} / \tau_{\mathrm{J}}^{\mathrm{eff}}>\tau_{\lambda} / \tau_{\mathrm{J}}$ for $\lambda>1.25 \mu \mathrm{m}$.

Predictions of $\tau^{\text {eff }}$ for a turbulent medium, as in Fig. 10, are much more uncertain because the radiative transfer is then sensitive to the clump distribution.

\subsection{One star in a homogeneous block}

Figure 13 displays four effective extinction curves towards a star in a homogeneous medium in the form $T_{\lambda}^{\text {eff }}=\tau_{\lambda}^{\text {eff }} / \tau_{\mathrm{V}}^{\text {eff }}$ (top) and $E_{\lambda}^{\text {eff }}$. The geometry is shown in Fig. $11 ; \omega_{\lambda}$ and $g_{\lambda}$ are from Fig. 6. Curves with other parameters look similar and one can, at least qualitatively, extrapolate their behavior from the plotted ones.

- In the two strings of red and green circles, the star is at the block center (Fig. 11a) and the angle $\Omega=90^{\circ}$. The visual extinction optical depth towards the star equals 4 (green) and 1 (red). The curve with $\tau_{\mathrm{V}}=0.5$ (not plotted) lies only marginally above the one with $\tau_{\mathrm{V}}=1$.

$\Omega=90^{\circ}$ implies that in an observation with 1 arcsec resolution towards a star (stars) in a galaxy at $D=20 \mathrm{Mpc}$ the linear scale of the aperture is $100 \mathrm{pc}$ (comparable to the scale height of a disk galaxy). Scattering is then negligible 


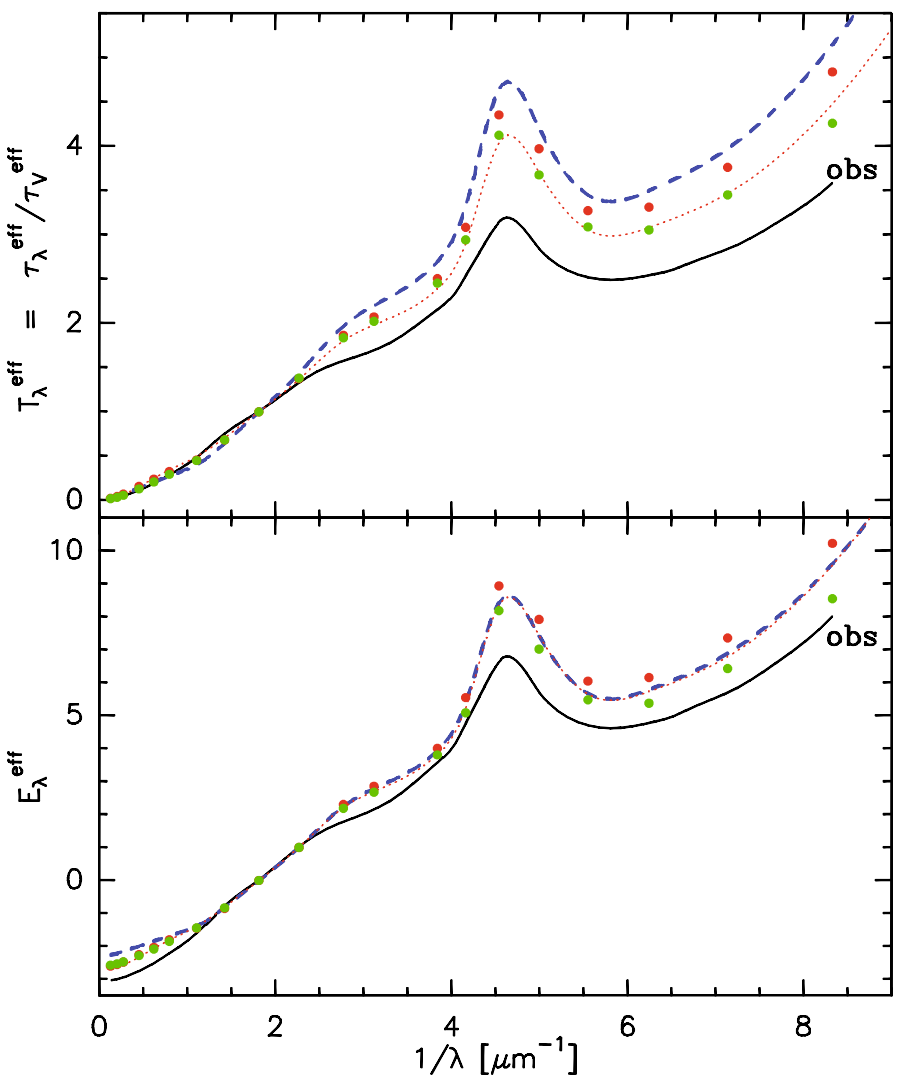

Fig. 13. Four effective extinction curves towards a star in a homogeneous block. Circles: star is in the cloud center (Fig. 11a) and $\Omega=90^{\circ}$; green: $\tau_{\mathrm{V}}=4$; red: $\tau_{\mathrm{V}}=1$. Dotted red line: $\Omega=90^{\circ}, \tau_{\mathrm{V}}=1$, but star is behind cloud (Fig. 11b). Blue dashes: $\tau_{\mathrm{V}}=2$, star is at cloud center, but $\Omega=127^{\circ}$. Solid line: standard reddening for comparison.

only if the mean scattering optical depth around the star over $\sim 100 \mathrm{pc}$ is small $(<0.1)$.

- At $\lambda<0.55 \mu \mathrm{m}$, the elevation above the fiducial standard curve (marked "obs") decreases as $\tau_{\mathrm{V}}$ increases.

- When $\Omega \rightarrow 0$ (not shown), $T_{\lambda}^{\mathrm{eff}} \rightarrow T_{\lambda}$ and $E_{\lambda}^{\mathrm{eff}} \rightarrow E_{\lambda}$. Deviations are always the result of insufficient spatial resolution.

- In the blue dashed curve, the star is also at the block center, $\tau_{\mathrm{V}}=2$, and the angle $\Omega$ is wider $\left(127^{\circ}\right)$, enhancing the scattered light.

- In the red dotted curve (which is indistiguishable from the blue dashes in the lower frame), $\tau_{\mathrm{V}}=1$ and $\Omega=90^{\circ}$, but the star is at the far side of the cloud (Fig. 11b). Photons emitted to the rear then cannot reach the observer and $T_{\lambda}^{\text {eff }}$ is reduced.

- At $\lambda>0.55 \mu \mathrm{m}, T_{\lambda}^{\mathrm{eff}}$ and $T_{\lambda}$ do not differ much. $E_{\lambda}^{\mathrm{eff}}$, on the other hand, lies noticably above $E_{\lambda}$ and therefore $R_{\mathrm{V}}^{\text {eff }}<$ $R_{\mathrm{V}}=3.1$.

\subsection{One star in a clumpy block}

When the medium is clumpy, the excursions of the departives extinction curves from standard reddening are qualitatively similar, but can be more pronounced at short $(\lambda<0.44 \mu \mathrm{m})$ as well as long $(\lambda>0.55 \mu \mathrm{m})$ wavelengths. Also the scatter between the curves is large. Four examples of clumpy models are drawn in Fig. 14 (four dotted colored lines). The star is always at the block center (Fig. 11a), $\Omega=90^{\circ}$ and $\tau_{\text {mean }}=1$. All clumps are identical and their positions are chosen randomly.
We restrict the further description of the models to the parameters: volume filling factor $f_{\text {Vol }}$, visual optical depth towards the star $\tau_{\mathrm{V}}$, and its value $\tau_{\text {mean }}$ if the medium were homogeneous while the total dust mass remained constant (see Sect. 6.3).

To identify the curves, we note that in the top frame, for $\lambda^{-1}>2 \mu \mathrm{m}^{-1}$, they have from top to bottom the parameters: $\left(\tau_{\mathrm{V}}, f_{\mathrm{Vol}}\right)=(0.34,0.09),(0.44,0.21),(0.58,0.21)$ and $(1.16$, 0.21 ). As benchmarks, we plot the standard reddening curve (labeled "obs") and from Fig. 13 the curve where the medium is uniform, the star is at the block center, $\tau_{\mathrm{V}}=1$ and $\Omega=90^{\circ}$ (red circles not connected by a line).

The extinction curves in Figs. 13 and 14 resemble, for $\lambda^{-1}>$ $2 \mu \mathrm{m}^{-1}$, those towards the Magellanic clouds (Prévot et al. 84; Gordon et al. 2003). They are themselves very diverse, but have in common a steep UV rise. The rough agreement can be further improved if one reduces the $4.6 \mu \mathrm{m}$ bump in the models by lowering the fraction of small graphitic particles.

The $R_{\mathrm{V}}^{\text {eff }}$-values in Figs. 13 and 14 are systematically below the standard $R_{\mathrm{V}}=3.1$ and can even become as small as one. Of course, it is clear from the definition in Eq. (8) that the model results for $R_{\mathrm{V}}^{\text {eff }}$ are sensitive to the albedo at $\mathrm{B}$ and $\mathrm{V}$ and to the asymmetry factor, both of which we do not know well. Nevertheless, our values of $R_{\mathrm{V}}^{\mathrm{eff}}$ in the figures are, at least, qualitatively in line with observations. Gordon et al. (2003) found in the Magellanic clouds towards weakly reddened stars $\left(E_{\mathrm{B}-\mathrm{V}}^{\mathrm{eff}} \simeq 0.2 \mathrm{mag}\right)$ an average $R_{\mathrm{V}}^{\text {eff }}$ of 2.75 together with some very small values $(\lesssim 2)$.

Clayton et al. (2000) and Weingartner \& Draine (2001) explain the anomalous SMC/LMC extinction curves by modifications of the grain size and the carbon abundance (note that the reduced dust-to-gas ratio in these clouds by itself does not affect $E_{\lambda}^{\text {eff }}$ ), whereas we point out that they may also result from scattering off standard dust.

\subsection{The effective standard color excess}

When scattered radiation is relevant, the standard color excess $E_{\mathrm{B}-\mathrm{V}}$ and the ratio of total-to-selective extinction, $R_{\mathrm{V}}$, have to be replaced by $E_{\mathrm{B}-\mathrm{V}}^{\mathrm{eff}}$ and $R_{\mathrm{V}}^{\mathrm{eff}}$, respectively. $E_{\mathrm{B}-\mathrm{V}}^{\mathrm{eff}}$ follows observationally in the usual way by comparing the reddened star with an unreddened object of the same spectral shape. To then determine $A_{\mathrm{V}}^{\mathrm{eff}}=1.086 \tau_{\mathrm{V}}^{\mathrm{eff}}$ from the relation

$A_{\mathrm{V}}^{\mathrm{eff}}=E_{\mathrm{B}-\mathrm{V}}^{\mathrm{eff}} R_{\mathrm{V}}^{\mathrm{eff}}$

requires knowledge of $R_{\mathrm{V}}^{\text {eff }}$ which is usually missing. The few examples in Figs. 13 and 14 suggest that $R_{\mathrm{V}}^{\text {eff }}$ may be smaller than $R_{\mathrm{V}}$ by about $10 \%$ for a homogeneous medium and by much more for a clumpy one. If one uses $R_{\mathrm{V}}$ instead of $R_{\mathrm{V}}^{\text {eff }}$ in Eq. (24), one tends to overestimate $\tau_{\mathrm{V}}^{\text {eff }}$ and thus the intrinsic stellar luminosity. $R_{\mathrm{V}}^{\text {eff }}$ has to be gathered in the usual way from infrared photometry.

\section{How to identify scattered light}

There are two indicators that a photometric measurement of a single star is contaminated by scattered photons. First, when repeating the observation at higher spatial resolution, the flux should go down. Second, the scattered light is polarized with the polarization vector perpendicular to the radial vector twards the star. This is well known from reflection nebulae.

To approximately quantify these effects, consider a star at a distance $D$, in a non-opaque $(\tau<1)$ homogeneous medium 


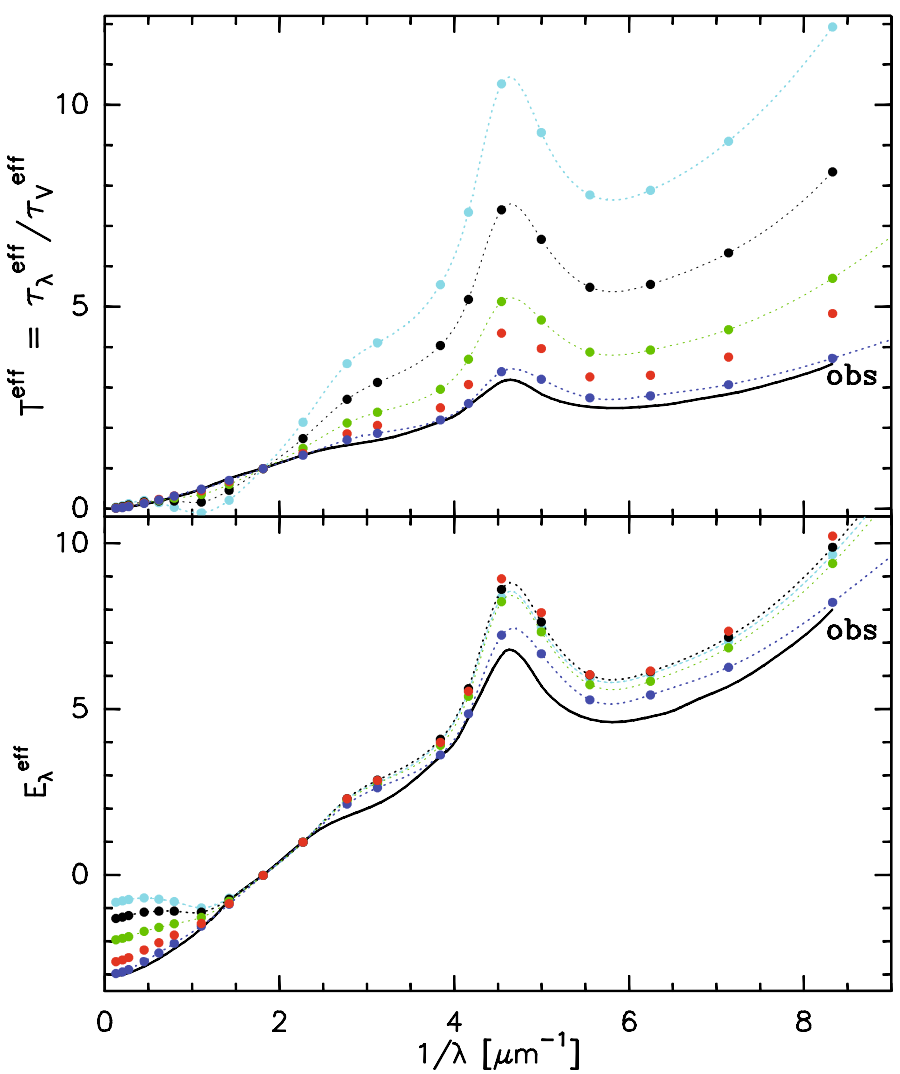

Fig. 14. Four effective extinction curves towards a star in a clumpy block, all with $\Omega=90^{\circ}$ (dotted lines with filled circles). For comparison: standard reddening (solid black line) and homogeneous dust with $\tau_{\mathrm{V}}=1$ (red circles, exactly as in Fig. 13). The total dust mass is the same in all distributions.

of hydrogen density $n$. Let $F^{*}$ be the flux that comes directly from the star and $F^{\text {sca }}$ the flux from scattered light only. The latter is proportional to the scattering optical depth, $\tau^{\text {sca }}$, within the aperture, $F^{\mathrm{sca}} \simeq \tau^{\mathrm{sca}} F^{*}$. Therefore, as the aperture decreases, the flux should drop by up to $\sim 100 \tau^{\text {sca }}$ percent. If $\varphi$ denotes the angle of the aperture and $\kappa^{\text {sca }}$ the scattering coefficient per $\mathrm{H}$ atom, $\tau^{\mathrm{sca}} \simeq \frac{1}{2} \varphi D n \kappa^{\mathrm{sca}}$.

As an example, consider the Magellanic clouds $(D=$ $50 \mathrm{kpc}$ ). The brightest stars there have an apparent visual magnitude $m_{\mathrm{V}} \simeq 12 \mathrm{mag}$. Assuming standard Milky Way dust, $\kappa^{\text {sca }} \simeq 2.5 \times 10^{-22} \mathrm{~cm}^{2}$ at V. In the IUE mission, $\varphi$ was about $2^{\prime \prime}$, for the HST, $\varphi \simeq 0.1^{\prime \prime}$ implying $\tau^{\text {sca }} \simeq 2 \times 10^{-4} n$ and $10^{-5} n$, respectively. Therefore, if the medium is of moderate density $\left(n=100 \ldots 1000 \mathrm{~cm}^{-3}\right)$, contamination of an IUE measurement by scattered light could easily be confirmed with the HST.

That it is really due to scattering and not to other sources, for instance, stars too weak to be seen individually, can be verified by measuring the polarization. When the aperture is centered on the star, the net polarization vanishes for reasons of symmetry. When the aperture is offset to a position where the star lies just outside $\left(F^{*}=0\right)$, one receives the flux $F^{\text {sca }}=f \tau^{\text {sca }} F^{*}$ where $f \sim \frac{1}{6}$ (when the aperture is centered on the star, $f=1$ ). The degree, $p$, to which $F^{\text {sca }}$ is polarized should be as high as in reflection nebulae $(p \gtrsim 30 \%)$. Polarization by dust near bright stars in the LMC/SMC, and even far beyond, should therefore also readily be detectable.

However, when the medium is not homogeneous, but peaked towards the star or when there is circumstellar material, the demands on the spatial resolution are, of course, much higher and may exceed present day technical capabilities.

Unfortunately, it is also not possible to identify, by means of polarization and aperture-dependence of the flux, scattered light from homogeneous disks of stars and dust (Sect. 6.2). The flux is then always proportional to the aperture area and the net polarization is always zero. An inhomogeneous distribution of stars or dust (Sect. 6.3) may, of course, lead to polarization, which would then naturally be attributed to reflection. But even with the best resolution, distant objects (galaxies) tend to become homogeneous on a scale of $100 \mathrm{pc}$.

\section{Comparison with previous computations}

The radiative transfer through a dusty medium, neglecting thermal grain emission, has been solved repeatedly (see references in Sect. 1) and the relevance of scattered light acknowledged. A detailed comparison of the literature results with ours is in the majority of cases quite cumbersome, however, in a few cases it is straightforward and we discuss them below.

\subsection{Spheres}

For homogeneous spheres with a single central star, Mathis (1972) presents in his Eq. (9) a general formula for $\tau^{\text {eff }}$ which includes the asymmetry factor $g$ :

$\mathrm{e}^{-\tau^{\mathrm{eff}}}=\mathrm{e}^{-\tau}+1.1 \omega\left[1-\mathrm{e}^{-\tau}\right] \mathrm{e}^{-\tau \sqrt{1-\omega}(1-g)^{0.3}}$.

It yields $\tau^{\text {eff }} \simeq \tau(1-1.1 \omega)$ for small $\tau$ and arbitrary $g$. When $\tau$ is large and $g=0$, one finds $\tau^{\text {eff }} \simeq \tau$ for $\omega=0$, and $\mathrm{e}^{-\tau^{\mathrm{eff}}}=1.1 \omega \mathrm{e}^{-\tau \sqrt{1-\omega}}$ for $\omega>0$. We plot an example of $\tau^{\mathrm{eff}} / \tau$ from Eq. (25) in Fig. 7. The agreement with our curve is tolerable (compare triangles and squares), but for certain sets of $(\omega, g)$, it is poor at high $\tau$.

When scattering is isotropic and $\tau$ large, Rybicki \& Lightman (1979) predict $\tau^{\text {eff }} / \tau \rightarrow \sqrt{1-\omega}$, but at least up to $\tau \simeq 150$, the radiative transfer values in Fig. 7 are considerably higher (see also Appendix A).

Witt et al. (1992) and Witt \& Gordon (2000) performed Monte Carlo calculations of dusty spheres filled uniformly with many stars. The medium may be clumpy or homogeneous. This scenario is considered by us only marginally in Eq. (11).

\subsection{Slabs}

With regard to slabs, we compare with Calzetti et al. (1994) because their paper is frequently used for photometric corrections. Their basic mathematical expressions for $\tau^{\mathrm{eff}}$ are taken from Natta \& Panagia (1984).

CKS consider in their Fig. 8 five scenarios which we will discuss consecutively. Let $L$ denote the extension of the stellar slab and the dust slab (screen) perpendicular to the line of sight, and $d$ the separation between the two. When $d \gg L$, almost no scattered light is detected. When $d \ll L$, the configuration can be approximated by $L=\infty$, as in our calculations for Figs. 8, 9 and 10, and the scattered light must be included.

1. The screen is uniform and distant $(d \gg L)$. This is the trivial case where $\tau^{\mathrm{eff}}=\tau$.

2. The screen is clumpy and distant $(d \gg L)$. Scattering is again irrelevant and the effective optical depth is for each star $i$ equal to its extinction optical depth: $\tau_{i}^{\text {eff }}=\tau_{i}$. For identical 
clumps of extinction optical depth $\tau_{\mathrm{c}}$ with a Poisson distribution, Natta \& Panagia (1984) found the analytical solution

$\tau^{\mathrm{eff}}=N\left[1-\mathrm{e}^{-\tau_{\mathrm{c}}}\right]$.

$N$ is the average number of clumps in the line of sight; the mean extinction optical depth is therefore $\tau_{\text {mean }}=N \tau_{\mathrm{c}}$. Note that $\tau^{\text {eff }}$ is not a function of $\tau_{\text {mean }}$, but depends on $N$ and $\tau_{\mathrm{c}}$ separately. We mention that Eq. (11) for the total effective optical depth, $\tau^{\text {eff }}$, also leads to Eq. (26) when summing over a Poisson distribution and putting $f_{i}=$ const.

3. When the dust screen is close $(d \ll L)$ and homogeneous and the scattering is isotropic, CKS use

$\tau^{\mathrm{eff}} / \tau \simeq \sqrt{1-\omega}$

following Natta \& Panagia (1984). The formula is attributed to Code (1973) and Mathis (1983), however, it is not stated in any of these papers explicitly. According to Eq. (27), $\tau^{\text {eff }} / \tau$ is constant whereas we find that it increases with $\tau$ (see Fig. 8a).

4. When the dust screen is close $(d \ll L)$ and clumpy, CKS propose

$\tau^{\mathrm{eff}}=N\left[1-\mathrm{e}^{-\tau_{\mathrm{c}} \sqrt{1-\omega}}\right]$,

which follows from Eq. (26) by replacing $\tau_{\mathrm{c}}$ with $\tau_{\mathrm{c}} \sqrt{1-\omega}$ to account for clumping (see Eq. (27)). Our calculations in Fig. 10 agree poorly with the approximation of Eq. (28).

5. When the emitters (stars) and absorbers (dust) are uniformly mixed $(d=0)$, CKS use

$$
\mathrm{e}^{-\tau^{\mathrm{eff}}}=\frac{1-\mathrm{e}^{-\tau \sqrt{1-\omega}}}{\tau \sqrt{1-\omega}},
$$

which follows from Eq. (20) by substituting $\tau \sqrt{1-\omega}$ for $\tau$. Our results in Fig. 9 are quite discrepant. For example, when $\omega=0.6, g=0$ and $\tau=1$, Eq. (29) yields $\tau^{\text {eff }}=0.3$ whereas we find $\tau^{\text {eff }} \simeq 0$. Our $\tau^{\text {eff }}$ implies that the scattered light fully compensates for the attenuation which the direct stellar light suffers along the line of sight.

Summarizing, there is disagreement in all scenarios (3 to 5) where scattered light is involved.

\section{Summary and concluding remarks}

In each photometric measurement of a star, a certain fraction, $f$, of the detected photons has been reflected by dust. Usually $f$ is extremely small and negligible, however, in some important cases, it is not negligible and then one needs the effective optical depth $\tau^{\text {eff }}{ }_{\lambda}$, and not the extinction optical depth $\tau_{\lambda}$, to deredden the source. The intrinsic luminosity at wavelength $\lambda$ is then given by $L_{\lambda}=4 \pi D^{2} F_{\lambda}^{\mathrm{obs}} \mathrm{e}_{\lambda}^{\tau_{\lambda}^{\mathrm{eff}}}$, where $D$ is the source distance and $F_{\lambda}^{\mathrm{obs}}$ the observed flux.

One must correct for scattered light whenever the scattering optical depth, $\tau^{\text {sca }}$, within the region covered by the aperture (see Fig. 1) is greater than relative observational error $\varepsilon_{\text {err }}$. This is the case in the photometry of stars with circumstellar shells, but also bright stars in another galaxy, distant star clusters, galactic nuclei and whole galaxies.

To quantify the necessary corrections, we computed $\tau^{\text {eff }}$ for a variety of idealized geometries (spheres, slabs, blocks; uniform or clumpy) varying the extinction optical depth, $\tau$, the albedo and the phase function. Even in the simplest scenario, a homogenous sphere with a central star, we did not find a handy formula to relate $\tau^{\text {eff }}$ to $\tau$. Full radiative transfer calculations always seem to be necessary.

Most calculations are summarized in figures which readily allow one to read off $\tau^{\text {eff }}$ or $\tau^{\text {eff }}{ }_{\lambda} / \tau_{\mathrm{V}}^{\text {eff }}$ (to obtain them in tabular form, please contact p309ekr@mpifr-bonn.mpg.de). The results for infinite slabs may, cum grano salis, be applicable to galactic disks, those for a dust block with a single star to any star with sufficient circumstellar matter in the aperture $\left(\tau^{\text {sca }} \gtrsim \varepsilon_{\text {err }}\right)$.

We did not consider star clusters explicitly because they are generally very complex, but some results for slabs may be carried over, at least qualitatively. The complexity of clusters does not pose a numerical problem (MC can handle almost anything), the difficulty lies in choosing for a specific astronomical source the right distribution of stars and dust.

We also present a few effective extinction curves assuming standard dust. They turn out to be sensitive to the spatial resolution and the structure of the medium (clumpiness, foreground and background column desities) demonstrating that distortions of the standard reddening curve may be caused not only by nonstandard grain properties, but also by reflected light.

Acknowledgements. I am much obliged to the referee, John Mathis, for pointing out in friendly words the limited astronomical relevance of the paper. I tried to improve but even with the kind help of Malcolm Walmsley who took a more lenient view, I may not have succeeded. My warm thanks also go to Werner Tscharnuter and Ralf Siebenmorgen for helpful comments.

\section{Appendix A: Limiting values of $\tau^{\text {eff }} / \tau$}

In Sect. 6, we computed for various configurations the ratio $\tau^{\text {eff }} / \tau$ in the range $0.01 \lesssim \tau \lesssim 100$. We now discuss the limiting behavior when scattering is isotropic.

\section{A.1. Small $\tau$}

- Sphere with central star (Sect. 6.1). A photon interacts at most once so that all scattered photons escape. The luminosity outside the sphere is then $L \mathrm{e}^{-\tau^{\text {abs }}}$ and the observed flux $F=F^{\text {nd }} \mathrm{e}^{-\tau^{\text {abs }}}$. Therefore,

$\tau^{\mathrm{eff}}=\tau(1-\omega)$

and the effective extinction curve becomes

$T_{\lambda}^{\mathrm{eff}}=\frac{\tau_{\lambda}^{\mathrm{eff}}}{\tau_{\mathrm{V}}^{\mathrm{eff}}}=\frac{\tau_{\lambda}^{\mathrm{abs}}}{\tau_{\mathrm{V}}^{\mathrm{eff}}}$.

- Star layer behind dust screen (Sect. 6.2.1). The observational aperture covers on the screen an area $A_{\text {ap }}=\pi a^{2} / 4$. If $N_{*}$ is the column density of the stars and each star has a luminosity $L$, without dust an observer would receive the flux $F^{\text {nd }}=$ $N_{*} L A_{\text {ap }} / 4 \pi D^{2}$. The screen weakens it by

$\Delta F_{1}=\tau F^{\text {nd }} ;$

but also strengthen it by scattering light from stars that lie mainly outside the observer's field of view. The stars in an annulus with radii $r$ and $r+d r$ around point $A$ in Fig. 4 have a luminosity $2 \pi r d r N_{*} L$ of which the fraction $A_{\text {ap }} \tau^{\text {sca }} / 4 \pi s^{2}$ is scattered within the aperture (the scattering optical thickness for light from $P^{\prime}$ is $\tau^{\text {sca }} s / d$ and the projected surface of the aperture seen from $P^{\prime}$ is $A_{\text {ap }} d / s$ ). With $s^{2}=d^{2}+r^{2}$, the total flux enhancement due to scattering is therefore

$\Delta F_{2}=\frac{N_{*} L A_{\mathrm{ap}} \tau^{\mathrm{sca}}}{8 \pi(D-d)^{2}} \int_{0}^{r_{u}} \frac{r \mathrm{~d} r}{d^{2}+r^{2}}$. 
The upper integration limit, $r_{u}$, is roughly set by the condition $\tau^{\text {abs }} \sqrt{r_{u}^{2}+d^{2}} / d=1$. The primitive of $r /\left(d^{2}+r^{2}\right)$ is $\frac{1}{2} \ln \left(d^{2}+r^{2}\right)$, the integral therefore equals $-\ln \tau^{\text {abs }}$ and

$\Delta F_{2}=-\frac{1}{2} F^{\mathrm{nd}} \omega \tau \ln \tau^{\mathrm{abs}}$

so that

$\tau^{\mathrm{eff}}=\frac{\Delta F_{1}-\Delta F_{2}}{F^{\mathrm{nd}}}=\tau\left[1+\frac{1}{2} \omega \ln \tau^{\mathrm{abs}}\right]$.

- Dust slab with star layer in the midplane (Sect. 6.2.2). The reinforcement by scattered light comes from dust in front of and behind the stars and is therefore twice as large as in Eq. (A.3); consequently $\Delta F_{2}=-F^{\text {nd }} \omega \tau \ln \tau^{\text {abs }}$ and

$\tau^{\mathrm{eff}}=\tau\left[1+\omega \ln \tau^{\mathrm{abs}}\right]$.

\section{A.2. Large $\tau$}

Analytical expressions for the limit $\tau \rightarrow \infty$ are of principle interest, although one cannot detect a star when it is highly obscured. Slabs and spheres may already have been treated rigorously in the literature, but the author is not aware of such calculations.

Rybicki \& Lightman (1979) present a high- $\tau$ estimate for spheres. They assume a constant photon step size $\ell$ equal to the inverse of the extinction coefficient per $\mathrm{cm}^{3}$,

$\ell=\frac{1}{K^{\mathrm{ext}}}$

As the probability of absorption at the end of each step equals $K^{\text {abs }} / K^{\text {ext }}$, a photon takes on average

$N=\frac{K^{\mathrm{ext}}}{K^{\mathrm{abs}}}$

steps before being destroyed. In a random walk, corresponding to $g=0$, the root mean square displacement from the star is then $\ell_{*}^{2}=N \ell^{2}$. If $R$ is the radius of the dust sphere and $\tau=R K^{\text {ext }}$ its extinction optical thickness, the emerging flux is reduced by a factor $\mathrm{e}^{-\tau^{\mathrm{eff}}}$ where

$\tau^{\mathrm{eff}} \simeq \frac{R}{l_{*}}=\sqrt{\tau^{\mathrm{abs}} \tau}=\tau \sqrt{1-\omega}$.

This formula can only be approximate. Among others, photons do not make steps of constant size. Although their mean free path $\langle\ell\rangle=1 / K^{\mathrm{ext}}$, the root mean square displacement of the locus of absorption from the origin is $\sqrt{\left\langle\ell_{*}^{2}\right\rangle}=\sqrt{2}\langle\ell\rangle$ because

$\left\langle\tau^{2}\right\rangle=\frac{\int_{0}^{\infty} \tau^{2} \mathrm{e}^{-\tau} \mathrm{d} \tau}{\int_{0}^{\infty} \mathrm{e}^{-\tau} \mathrm{d} \tau}=2$,

with $\int_{0}^{\infty} \mathrm{e}^{-\tau} \mathrm{d} \tau=1$, whereas $\langle\tau\rangle=1$.

\section{References}

Bruzual, G., Magris, G., \& Calvet, N. 1988, ApJ, 333, 673

Calzetti, D., Kinney, A., \& Storchi-Bergmann, T. 1994, ApJ, 429, 582 (CKS)

Caplan, J., \& Deharveng, L. 1986, A\&A, 155, 297

Clayton, G. C., Wolff, M. J., Gordon, K. D., \& Misselt, K. A. 2000, ed. M. L. Sitko, A. L. Sprague, \& D. K. Lynch, ASP Conf. Ser., 196, 41

Code, A. D. 1973, IAU Symp., 52, 505

Fischera, J., \& Dopita, M. 2005, ApJ, 619, 340

Gordon, K. D. 2004, ASP Conf. Ser. 309, ed. A. N., Witt, G. C. Clayton, \& B. T. Draine, 77

Gordon, K. D., Clayton, G. C., Misselt, K. A., \& Landolt, A. U., \& M. J. Wolff, 2003, ApJ, 594, 279

Greenberg, J. M., \& Wang, R. T. 1972, Mem. Soc. Roy. Sci. Liege, Ser. 6, 3, 197 Henyey, L. G., \& Greenstein, J. L. 1941, ApJ, 93, 70

Krügel, E. 2006, An Introduction to the Physics of Interstellar Dust (Taylor \& Francis)

Mathis, J. M. 1972, ApJ, 176, 651

Mathis, J. M. 1983, ApJ, 267, 119

Natta, A., \& Panagia, N. 1984, ApJ, 287, 228

Prévot, M. L., Lequeux, J., Maurice, E., Prévot, L., \& Rocca-Volmerange B., 1984, A\&A, 132, 389

Rybicki, G. B., \& Lightman, A. P. 1979, Radiative Processes in Astrophysics, 36ff. (John Wiley)

Voshchinnikov, N. V., Molster, F. J., \& Thé, P. S. 1996, A\&A, 312, 243

Weingartner, J. C., \& Draine, B. T. 2001, ApJ, 548, 296

Whittet, D. C. B. 2003, Dust in the Galactic Environment (IoP)

Witt, A. N., \& Gordon, K. D. 2000, ApJ, 528, 799

Witt, A. N., Thronson, H. A., \& Capuano, J. M. 1992, ApJ, 393, 611 